\title{
Parametrizations for Integral Slope Homogeneous Modules over Tubular Canonical Algebras
}

\author{
Piotr Dowbor · Hagen Meltzer • Andrzej Mróz
}

Received: 6 December 2011 / Accepted: 17 October 2012 / Published online: 9 December 2012 (C) The Author(s) 2012. This article is published with open access at Springerlink.com

\begin{abstract}
We give a description of matrix bimodules parametrizing all indecomposable homogeneous $\Lambda$-modules with a fixed integral slope over a tubular canonical algebra $\Lambda$, for all possible integers (Theorem 4.1). An important role in the first step of this description (Theorem 2.4) is played by the translation of the shift functor for coherent sheaves over the associated weighted projective line to the language of $\Lambda$ modules (Theorem 3.2).
\end{abstract}

Keywords Parametrizing bimodule $\cdot$ Homogeneous module $\cdot$ Canonical tubular algebra $\cdot$ Weighted projective line $\cdot$ Coherent sheaf $\cdot$ Derived category $\cdot \xi$-matrix $•$ Quasi-diagonal form

Mathematics Subject Classifications (2010) $16 \mathrm{G} 20 \cdot 16 \mathrm{G} 60 \cdot 16 \mathrm{G} 70 \cdot 14 \mathrm{~F} 05$

\section{Introduction}

The class of canonical algebras, introduced and studied intensively by Ringel [18], is one of the most significant and well understood from the point of view of the representation theory class of finite dimensional algebras over a field. Especially interesting are tubular canonical algebras, which yield a model example of tame algebras having non-domestic, but polynomial growth representation type.

The structure of the category $\bmod \Lambda$ of finite dimension right $\Lambda$-modules for a tubular canonical $k$-algebra $\Lambda$ is well described. It is in a certain sense a result of

Presented by Alain Verschoren.

Piotr Dowbor and Andrzej Mróz are supported by the Research Grant N N201 542138 of the Polish Ministry of Science and Higher Education.

P. Dowbor $(\varangle) \cdot$ A. Mróz

Faculty of Mathematics and Computer Science, Nicolaus Copernicus University, Chopina 12/18, 87-100 Toruń, Poland

e-mail: dowbor@mat.uni.torun.pl

A. Mróz

e-mail: amroz@mat.uni.torun.pl

H. Meltzer

Institute of Mathematics, Szczecin University, Wielkopolska 15, 70-451 Szczecin, Poland e-mail: meltzer@wmf.univ.szczecin.pl 
the fact that $\bmod \Lambda$ is controlled on the level of the Grothendieck group $\mathrm{K}_{0}(\Lambda)$ by the Euler form $\chi_{\Lambda}$ of $\Lambda$, in terms expressed by the so called separation property. In particular, full information on the shape of the Auslander-Reiten quiver $\Gamma_{\Lambda}$ is available, with a precise description of its connected components: the preprojective and preinjectitive one, as well as the collection of $\mathbb{P}^{1}(k)$-families of tubes indexed by the set $\overline{\mathbb{Q}}_{0}^{\infty}:=\mathbb{Q}_{\geq 0} \cup\{\infty\}$. In this sense, the classification of all indecomposable $\Lambda$-modules, up to isomorphism, referring to their dimension vectors and "tubular coordinates" is known since many years. Nevertheless, it is not so satisfactory, as could be an eventual construction of a complete list of matrix presentations representing all isomorphism classes of indecomposable $\Lambda$-modules. Such a list is not known up to now and its creation remains one of the very last important open questions concerning representation theory of tubular canonical algebras. Notice that actually there was even no possibility of an effective direct verification of the definition of tameness for $\Lambda$ (see e.g. [19], cf. also Section 2.3). On the other hand the existence of such a list would have an essential meaning for studying other challenging open problems of representation theory for $\Lambda$ like determining the multiplicity vectors or isomorphism question (see [4-6]).

To give an explicit matrix description of all indecomposable modules over a tame algebra, even in the case the dimension vectors of the indecomposables are known, is usually not an easy task. Nevertheless, for the case of domestic canonical algebras, i.e. those of Euclidean type, this problem was completely solved in [15]. In the last years also for the tubular case a remarkable progress has been achieved. Recently, a nontrivial effective algorithm for creating matrix presentations for indecomposables from the exceptional tubes over a tubular canonical algebra $\Lambda$ was discovered (see [8], also [17]). There were obtained also some results [13], which allow to find matrix presentations of preprojective and preinjective indecomposables over the corresponding hereditary algebra of Euclidean type. In this paper we study the problem of constructing matrix presentations for the remaining regular indecomposable $\Lambda$ modules, namely, these from homogeneous tubes. It is clear that this problem is fundamental in context of the verification of tameness for $\Lambda$.

More precisely, we want to construct for each index $\gamma \in \overline{\mathbb{Q}}_{0}^{\infty}$, a matrix bimodule $k[\xi]_{f} B_{\Lambda}\left(={ }_{k[\xi]_{f}} B_{\Lambda}(\gamma)\right)$, which yields a parametrization (by means of a tensor product) of all indecomposable $\Lambda$-modules in homogeneous tubes from the $\mathbb{P}^{1}(k)$-family $\mathcal{T}_{(\gamma)}$ by indecomposable $k[\xi]_{f}$-modules, where $k[\xi]_{f}$ is the localization of the polynomial algebra $k[\xi]$ in one variable $\xi$, defined by the polynomial $f \in k[\xi]$. Observe that once we know $B(\gamma)$, then for any pair $(c, l) \in(k \backslash \mathcal{Z}(f)) \times \mathbb{N}_{\geq 1}$, replacing in the $k[\xi]_{f}$-matrices forming $B(\gamma)$ the scalars $a$ by the scalar matrices $a \cdot I_{l}$ and $\xi$ by the Jordan blocks $J_{l}(c)$, we obtain the matrix $\Lambda$-module representing the indecomposable homogeneous module $\mathcal{H}_{\gamma}(c, l)$ of index $\gamma$ and quasi-length $l$, over the point $c \in \mathbb{P}^{1}(k)$.

In this article we present the solution of this problem for all $\gamma \in \overline{\mathbb{Q}}_{0}^{\infty}$ such that the slope $q=q(\gamma)$ associated to $\gamma$ is an integer (see Sections 2.1 and 2.2 for the definition). In Theorem 4.1 we give the precise matrix description of parametrizing bimodules as above, for all integral slopes (see Section 4.7).

The solution consists of two steps. In the first one, for each tubular case we find a common universal presentation for all modules $\mathcal{H}_{0}(c, l$ ) (they have slope $p$ and their matrix forms are well known since they are just homogeneous modules over a hereditary algebra of Euclidean type, see Section 2.1 for the definition of $p$ for $\Lambda)$. Next we transform it by some functors $\tilde{\Delta}_{(n)}, n \in \mathbb{N}$, referring to the structure on $\bmod \Lambda$, which is not visible from the level of $\Lambda$ and goes back to the Geigle- 
Lenzing approach of studying the module categories over canonical algebras in terms of the categories of graded coherent sheaves over the weighted projective lines $\mathbb{X}$ associated to $\Lambda$, more precisely, via the equivalence $\mathcal{D}^{b}(\bmod \Lambda) \simeq \mathcal{D}^{b}(\operatorname{coh} \mathbb{X})$ of the respective bounded derived categories [10]. Due to the connection to the shift by the twisted structure sheaf $\mathcal{O}_{\mathbb{X}}\left(\vec{x}_{t}\right)$, the functors $\tilde{\Delta}_{(n)}$ have nice properties (see Theorem 3.2) and allow to construct very precisely described presentations, by the $k[\xi] \otimes_{k} \Lambda$-modules being finitely generated free over $k[\xi]$, of the bimodules $C^{(n)}$ parametrizing all homogeneous indecomposable $\Lambda$-modules with the slope $n+p$, (see Theorem 2.4). In the second step, the most important and much more technically complicated step, we compute matrix forms of the bimodules $C^{(n)}$ applying a very concrete specialization of a quite general method formulated in Proposition 4.3. We use for this aim certain not completely trivial computer algebra facts concerning matrix calculus over $k[\xi]$ (see Section 4.5), which allow to reduce the amount of calculations for each tubular type to a finite number of cases and to explain precisely appearance of the formulas from Section 4.7.

The solution of the discussed problem for the remaining slopes has a different character and will be given in the subsequent publication (Dowbor, P., Meltzer, H., Mróz, A.: An algorithm for the construction of parametrizing bimodules for homogeneous modules over tubular canonical algebras. Preprint, 45pp. (2012), submitted).

In the paper we use standard definitions and notation which are well known and commonly used. For example, we denote by $\mathbb{N}$ (resp. $\mathbb{N}_{\geq m}$ ) the set of all natural numbers with 0 (resp. natural numbers greater or equal $m$ ). We use the similar notation in case of the sets $\mathbb{Z}$ and $\mathbb{Q}$ consisting of all integers and rational numbers, respectively. Moreover, for $n \in \mathbb{N}_{\geq 1}$ we set $[n]:=\{1, \ldots, n\}$. For the benefit of the reader, we briefly recall the most important basic notions in the consecutive sections. For other information concerning representation theory of algebras (respectively, derived categories, linear algebra) we refer to [1] (respectively, [11, 12], [9, 14]).

A field $k$ is usually assumed to be algebraically closed. For any polynomial $f \in$ $k[\xi]$ in one variable $\xi$ its zero set is always denoted by $\mathcal{Z}(f)$.

\section{Modules over Tubular Canonical Algebras}

\section{1}

Let $k$ be a field. By a canonical algebra we mean a canonical algebra of quiver type over $k$ in the sense of [18]. Such an algebra is given, up to isomorphism, as a quotient $k Q_{\mathbf{p}} / I_{\mathbf{p}, \lambda}$, where $Q_{\mathbf{p}}$ is the quiver

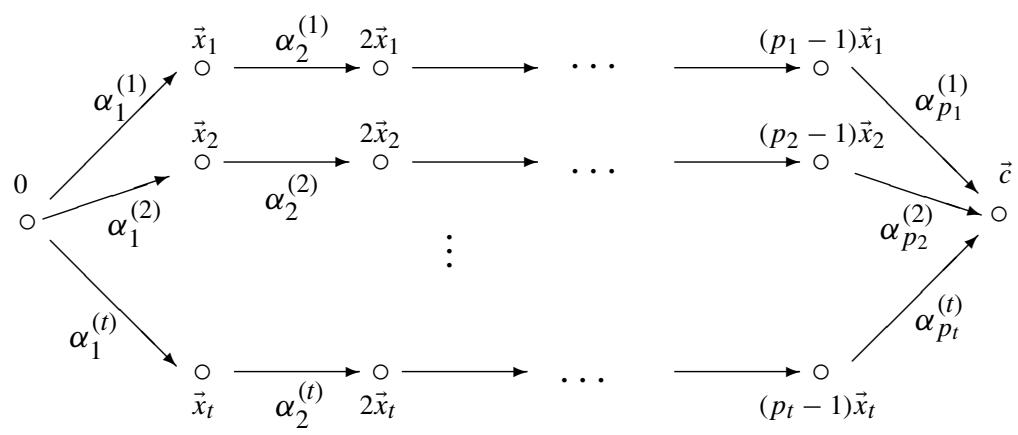


and $I_{\mathbf{p}, \lambda}$ is the ideal in the path algebra $k Q_{\mathbf{p}}$ generated by all elements $\alpha_{p_{i}}^{(i)} \cdots \alpha_{2}^{(i)}$ $\alpha_{1}^{(i)}-\alpha_{p_{1}}^{(1)} \cdots \alpha_{2}^{(1)} \alpha_{1}^{(1)}-\lambda_{i} \alpha_{p_{2}}^{(2)} \cdots \alpha_{2}^{(2)} \alpha_{1}^{(2)}, i=3, \ldots, t$, where the $\lambda_{i}$ are pairwise distinct non-zero elements of $k$. Thus $\Lambda$ depends on a sequence of natural numbers $\mathbf{p}=$ $\left(p_{1}, p_{2}, \ldots, p_{t}\right)$ and a sequence of parameters $\lambda=\left(\lambda_{3}, \ldots, \lambda_{t}\right)$. Recall that $\Lambda=$ $\Lambda(\mathbf{p}, \lambda)$ is called a tubular canonical algebra if and only if the sequence $\mathbf{p}$ is, up to permutation, one of the following $(2,2,2,2),(3,3,3),(2,4,4)$ or $(2,3,6)$. Without loss of generality one can assume that $\lambda_{3}=1$ and then, in case $t=3$, we simply write $\Lambda$ (p) assuming that $p_{1} \leq p_{2} \leq p_{3}$. A tubular canonical algebra $\Lambda$ of type $\mathbf{p}=(2,2,2,2)$ depends on one parameter $\lambda=\lambda_{4}$ and depending on the context we write $\Lambda((2,2,2,2))$ or $\Lambda((2,2,2,2), \lambda)$.

We denote by $\Lambda_{0}$ (respectively, $\Lambda_{\infty} \cong \Lambda_{0}^{o p}$ ) the hereditary algebra which is obtained from $k Q_{\mathbf{p}}$ by deleting the vertex $\vec{c}$ (respectively, 0 ).

Let $\bmod \Lambda$ be the category of finite-dimensional right $\Lambda$-modules, where $\Lambda=$ $\Lambda(\mathbf{p}, \lambda)$. We denote by $Q_{0}$ the set of vertices and by $Q_{1}$ the set of arrows of $Q_{\mathbf{p}}$. A finite-dimensional $\Lambda$-module $M$ is described by finite-dimensional vector spaces $M_{i}$ for each vertex $i$ of $Q_{0}$ and by linear maps $M_{\alpha}: M_{j} \rightarrow M_{i}$ for each arrow $\alpha: i \rightarrow j$ of $Q_{1}$ such that the relations of the ideal $I$ are satisfied by the linear maps. The collections $M=\left(\left\{M_{i}\right\}_{i \in Q_{0}},\left\{M_{\alpha}\right\}_{\alpha \in Q_{1}}\right)$ as above are usually called representations of $\Lambda$.

In this paper we consider also matrix representations of $\Lambda$, i.e. representations $M$ for which $M_{i}=k^{n_{i}}$ for each $i \in Q_{0}$ and $M_{\alpha}=A_{\alpha} \cdot: k^{n_{j}} \rightarrow k^{n_{i}}$, where $A_{i} \in \mathbb{M}_{n_{i} \times n_{j}}(k)$ for $\alpha: i \rightarrow j$ of $Q_{1}$ (we identify then $M_{\alpha}$ with $A_{\alpha}$, so $M$ is in fact a collection of matrices of suitable sizes). Note that the full subcategory $\bmod ^{\text {mat }} \Lambda$, formed by all matrix representations of $\Lambda$, is a dense subcategory in $\bmod \Lambda$.

For a representation $M$ we always denote by $\underline{\operatorname{dim}}(M)$ the vector $\left(\operatorname{dim}_{k}\left(M_{i}\right)\right)_{i \in Q_{0}} \in$ $\mathbb{N}^{Q_{0}}$, called the dimension vector of $M$. To $M$ we can associate two integers, its rank and degree, given by the formulas

$$
\begin{gathered}
\operatorname{rk}(M):=\operatorname{dim}_{k} M_{0}-\operatorname{dim}_{k} M_{\vec{c}} \\
\operatorname{deg}(M):=\sum_{i=1}^{t} \frac{p}{p_{i}}\left(\sum_{j=1}^{p_{i}-1} \operatorname{dim}_{k} M_{j \vec{x}_{i}}\right)-p \cdot \operatorname{dim}_{k} M_{\vec{c}}
\end{gathered}
$$

where $p$ denotes the least common multiple of the numbers $p_{1}, \ldots, p_{t}$ (note that $p=$ $p_{t}$, if $\mathbf{p}$ is of tubular type). They give rise to two linear forms rk, deg $: K_{0}(\Lambda) \rightarrow \mathbb{Z}$. For an indecomposable $\Lambda$-module $M$ we define also its slope $\mu(M):=\frac{\operatorname{deg}(M)}{\operatorname{rk}(M)} \in \overline{\mathbb{Q}}$, where $\overline{\mathbb{Q}}=\mathbb{Q} \cup\{\infty\}$. (Clearly, the value of $\mu(M)$ depends only on $\underline{\operatorname{dim}}(M)$ ).

For any $q \in \overline{\mathbb{Q}}$, we denote by $\mathcal{X}_{q}$ the full subcategory of $\bmod \bar{\Lambda}$ formed by all indecomposable $\Lambda$-modules with $\mu(M)=q$.

Let $R$ be a commutative $k$-algebra. Then by $\bmod R \otimes_{k} \Lambda$ we denote the category of all $R$ - $\Lambda$-bimodules being finitely generated as $R$-modules. Note that any such a bimodule $M$ can be interpreted as a representation $M=\left(\left\{M_{i}\right\}_{i \in Q_{0}},\left\{M_{\alpha}\right\}_{\alpha \in Q_{1}}\right)$ of $\Lambda$ such that all $M_{i}$ are finitely generated $R$-modules and all $M_{\alpha}$ are $R$-homomorphisms. We will consider two full subcategories of $\bmod R \otimes_{k} \Lambda$ : the subcategory $\bmod _{R} \Lambda$ formed by all $M$ such that $M_{i}$ is a free $R$-module for every $i \in Q_{0}$, and its dense 
subcategory $\bmod _{R}^{\text {mat }} \Lambda$ formed by all $M$ such that $M_{i}=R^{n_{i}}$ for every $i \in Q_{0}$, where $n_{i} \in \mathbb{N}$ (then $M_{\alpha}=A_{\alpha}: R^{n_{j}} \rightarrow R^{n_{i}}$, where $A_{i} \in \mathbb{M}_{n_{i} \times n_{j}}(R)$, for $\alpha: i \rightarrow j$ of $\left.Q_{1}\right)$.

For $M$ in $\bmod _{R} \Lambda$, by rank vector of $M$ we mean the vector $\underline{\operatorname{rk}}(M)=\left(\operatorname{rk}_{R}\left(M_{i}\right)\right) \in$ $\mathbb{N}^{Q_{0}}$, where $\operatorname{rk}_{R}\left(M_{i}\right)$ denotes the rank of a free $R$-module $\bar{M}_{i}$, for every $i \in Q_{0}$. Clearly, if $R=k[\xi]$ then $M$ from $\bmod _{R}^{\text {mat }} \Lambda$ can be treated as a collection $\left(A_{\alpha}\right)_{\alpha}$ of $\xi$-matrices (i.e. matrices in coefficients in $k[\xi]$ ) of suitable sizes determined by $\underline{\mathrm{rk}}(M)$.

Let $\Lambda$ be a canonical algebra of tubular type. Then the structure of the AuslanderReiten quiver $\Gamma_{\Lambda}$ of $\bmod \Lambda$, in particular the shape of its components, is well known [18] and it looks as follows:

$$
\Gamma_{\Lambda}=\mathcal{P} \sqcup\left(\coprod_{\gamma \in \overline{\mathbb{Q}}_{0}^{\infty}} \mathcal{T}_{(\gamma)}\right) \sqcup \mathcal{Q}
$$

where

- $\quad \mathcal{P}$ is a preprojective component which coincides with the preprojective component of $\bmod \Lambda_{0}$,

- $\quad\left(\mathcal{T}_{(\gamma)}\right)_{\gamma \in \overline{\mathbb{Q}}_{0}^{\infty}}$ consists of so-called separating $\mathbb{P}^{1}(k)$-families of tubes, all but $\mathcal{T}_{(0)}$ and $\mathcal{T}_{(\infty)}$ consisting only of stable ones (i.e. not containing a projective or injective module) of type $\mathbf{p}$,

- $\mathcal{Q}$ is a preinjective component which coincides with the preinjective component of $\bmod \Lambda_{\infty}$.

(Here $\overline{\mathbb{Q}}_{a}^{b}:=\overline{\mathbb{Q}}_{\leq b} \cap \overline{\mathbb{Q}}_{\geq a}=\{q \in \overline{\mathbb{Q}}: a \leq q \leq b\}$, for $\left.a, b \in \overline{\mathbb{Q}}\right)$.

Recall, that for a fixed $\gamma=\frac{\gamma_{\infty}}{\gamma_{0}} \in \overline{\mathbb{Q}}_{0}^{\infty}$ all dimension vectors of the $\Lambda$-modules from tubes in the family $\mathcal{T}_{(\gamma)}$ have index $\gamma$ (see [18] for the precise definition). Moreover, for each homogeneous tube they form the set $\mathbb{N}_{\geq 1} \cdot \bar{h}_{\gamma}$, where $\bar{h}_{\gamma}=\frac{1}{g} h_{\gamma}$ with $h_{\gamma}=$ $\gamma_{0} h_{0}+\gamma_{\infty} h_{\infty}$ and $g=\operatorname{gcd}\left\{\left(h_{\gamma}\right)_{i}: i \in Q_{0}\right\}$ (here $h_{0}, h_{\infty} \in \mathbb{Z}^{Q_{0}}$ denote the standard generators of radical spaces for the hereditary algebras $\Lambda_{0}$ and $\Lambda_{\infty}$, respectively; we assume always that $\gamma_{\infty}, \gamma_{0} \in \mathbb{N}$ are coprime).

Let now $q \in \overline{\mathbb{Q}}$. It is well known that if $p \leq q<\infty$ (respectively, $q \leq 0$ ) then $\mathcal{X}_{q}$ coincides with the full subcategory $\left\langle\mathcal{I}_{(\gamma)}\right\rangle$ of $\bmod \Lambda$ formed by all indecomposable modules isomorphic to those belonging to the family $\mathcal{T}_{(\gamma)}$ of tubes, where $\gamma(=$ $\gamma(q))=\frac{\gamma_{\infty}}{\gamma_{0}}$ is determined for $q$ by the equality $q=\mu\left(h_{\gamma}\right)$ (see $[10,16,18]$ and also [8] for details). Note that then $q=q(\gamma)$, where $q(\gamma):=p \frac{\gamma_{0}}{\gamma_{0}-\gamma_{\infty}}$, since $\operatorname{deg}\left(h_{0}\right)=$ $p^{2}, \operatorname{deg}\left(h_{\infty}\right)=0$ and $\operatorname{rk}\left(h_{\gamma}\right)=p\left(\gamma_{0}-\gamma_{\infty}\right)$ (case by case inspection). The rational number $q(\gamma)$ as above is called a slope associated to $\gamma$.

Now elementary computations show the following:

Lemma The mapping $\gamma \mapsto q(\gamma)$ and its inverse $q \mapsto \gamma(q)$ given for $q=\frac{a}{b}$ with $a \in \mathbb{N}, b \in \mathbb{Z}$ coprime, by the formula $\gamma(q)=\frac{\gamma_{\infty}}{\gamma_{0}}$, where $\gamma_{0}:=a$ and $\gamma_{\infty}:=a-$ 
$p b\left(\geq 0\right.$ !), yield a bijection $\overline{\mathbb{Q}}_{0}^{\infty} \longleftrightarrow \overline{\mathbb{Q}}^{\prime}:=\left(\overline{\mathbb{Q}} \backslash \mathbb{Q}_{0}^{p}\right) \cup\{0, p\}$. In particular, for the integers $q=p, p+1, p+2, \ldots, p+m, \ldots($ resp. $q=0,-1,-2, \ldots,-m, \ldots)$ we have $\gamma(q)=0,1-\frac{p}{p+1}, 1-\frac{p}{p+2}, \ldots, 1-\frac{p}{p+m}, \ldots$ (resp. $\infty, 1+p, 1+\frac{p}{2}, \ldots, 1+$ $\left.\frac{p}{m}, \ldots\right)$, and $\gamma(\infty)=1$.

Observe that therefore $\mathcal{X}_{p}=<\mathcal{T}_{(0)}>, \mathcal{X}_{\infty}=<\mathcal{T}_{(1)}>$ and $\mathcal{X}_{0}=<\mathcal{T}_{(\infty)}>$.

\section{3}

Let $\Lambda=k Q / I$ be a finite dimensional algebra defined by the bound quiver $(Q, I)$, where $Q=\left(Q_{0}, Q_{1}\right)$. It is clear that for such $\Lambda$, in the classical definition of tameness (see [7] and [19]) one can replace the dimensions of modules by their dimension vectors. Below we reformulate the known result, characterizing tameness of $\Lambda$ in terms of bimodules, which yield parametrizations not only of the $\Lambda$-modules in a fixed dimension vector (by simple modules over a localization $A$ of $k[\xi]$ ) but all $\Lambda$-modules belonging to the whole family of homogeneous tubes in the AuslanderReiten quiver of $\Lambda$, simultaneously in all dimension vectors being the multiplicities of the rank vector of this bimodule (now by indecomposable $A$-modules).

For a pair $d, d^{\prime} \in \mathbb{N}^{Q_{0}}$ of vectors we say that $d$ divides $d^{\prime}$ (and write $d \mid d^{\prime}$ ), if $d^{\prime}=l \cdot d$, for some $l \in \mathbb{N}$; then the unique such $l$ is denoted by $\frac{d^{\prime}}{d}$.

Theorem $A$-algebra $\Lambda$, as above, has tame representation type if and only if there exists a family $B^{(v)}, v \in \Omega$, of $A_{v}$ - $\Lambda$-bimodules, where $A_{v}:=k[\xi]_{f_{v}}$ for some $f_{v} \in$ $k[\xi]$, such that the left modules $A_{v} B^{(v)}$ are finitely generated free (with rank vector denoted by $r^{(v)} \in \mathbb{N}^{Q_{0}}$ ), satisfying the following two conditions:

- the functors $-\otimes_{A_{v}} B^{(v)}: \bmod A_{v} \rightarrow \bmod \Lambda$, for $v \in \Omega$, induce an injection between the sets of isoclasses of indecomposable modules and preserve AuslanderReiten sequences,

- for every $d \in \mathbb{N}^{Q_{0}}$, the set $\Omega(d):=\left\{v \in \Omega: r^{(v)} \mid d\right\}$ is finite and has the property that for a subset $\Omega^{\prime} \subseteq \Omega(d)$, the union

$$
\bigcup_{v \in \Omega^{\prime}}\left\{A_{v} /\left((\xi-c)^{\frac{d}{r^{(v)}}}\right) \otimes_{A_{v}} B^{(v)}: c \in k \backslash \mathcal{Z}\left(f_{v}\right)\right\}
$$

meets all but finitely many isoclasses of indecomposable $\Lambda$-modules of dimension vector $d$ if and only if $\Omega^{\prime}=\Omega(d)$.

(Note that $\Omega=\bigcup_{d \in \mathbb{N} Q_{0}} \Omega(d)$ and $\Omega(d) \subseteq \Omega\left(d^{\prime}\right)$, if $\left.d \mid d^{\prime}\right)$.

Proof The easier implication follows immediately by [7], the main one by the results from [2].

The bimodules $B^{(v)}, v \in \Omega$, satisfying the conditions above are called parametrizing bimodules for all indecomposable $\Lambda$-modules from the family of 
homogeneous tubes. (Observe that for a fixed $v$, the modules $A_{v} /\left((\xi-c)^{l}\right) \otimes_{A_{\nu}} B^{(v)}$, for $\left\{(c, l) \in k \backslash \mathcal{Z}\left(f_{v}\right)\right\} \times \mathbb{N}_{\geq 1}$, form a 1-parameter family of homogeneous tubes in $\bmod \Lambda$ consisting of modules with dimension vectors in $\left.\mathbb{N}_{\geq 1} \cdot r^{(v)}\right)$.

Let now $\Lambda=\Lambda(\mathbf{p}, \lambda)$ be a tubular canonical $k$-algebra of type $\mathbf{p}$. Then the very good knowledge on the structure of the category $\bmod \Lambda$ uniquely indicates that $\Lambda$ should have tame representation type, however, the precise description of the bimodules $B^{(v)}, v \in \Omega$, parametrizing the indecomposables in all homogeneous tubes is not known! It is clear that $\Omega$ has to be equal to $\overline{\mathbb{Q}}_{0}^{\infty}$, so by Lemma 2.2 we can assume that $\Omega=\overline{\mathbb{Q}}^{\prime}$. Moreover, $r^{(q)}=\bar{h}_{\gamma(q)}$, where $r^{(q)}$ is the rank vector of $B^{(q)}$ for $q \in \overline{\mathbb{Q}}^{\prime}$. Finally, for any $d \in \mathbb{N}^{Q_{0}}$ we have $\Omega(d)=\left\{B^{(q)}\right\}$, if $d \in \mathbb{N}_{\geq 1} \cdot r^{(q)}$ for some $q$; otherwise, $\Omega(d)=\emptyset$.

In this paper we give precise formulas for the bimodules $B^{(q)}$, for all $q \in \overline{\mathbb{Q}}^{\prime} \cap \mathbb{Z}$.

Let $\Lambda=\Lambda(\mathbf{p}, \lambda)$ be a canonical $k$-algebra. Then we can associate to $\Lambda$ the commutative $k$-algebra

$$
S=S(\mathbf{p}, \lambda):=k\left[X_{1}, \ldots, X_{t}\right] /\left(\left\{X_{i}^{p_{i}}-X_{1}^{p_{1}}-\lambda_{i} X_{2}^{p_{2}}\right\}_{i=3, \ldots, t}\right)
$$

$S$ admits a natural grading $S=\bigoplus_{\vec{x} \in \mathbf{L}} S_{\vec{x}}$ by the rank one abelian group $\mathbf{L}=\mathbf{L}(\mathbf{p})$ on generators $\vec{x}_{1}, \vec{x}_{2}, \ldots, \vec{x}_{t}$ with relations $p_{1} \vec{x}_{1}=p_{2} \vec{x}_{2}=\cdots=p_{t} \vec{x}_{t}=: \vec{c}$, such that the degree of each $X_{i}$ is just $\vec{x}_{i}$. $\mathbf{L}$ is an ordered group with $\mathbf{L}_{+}:=\sum_{i=1}^{t} \mathbb{N}_{i}$ being its set of positive elements and in fact $S_{\vec{x}} \neq 0$ if and only if $\vec{x} \in \mathbf{L}_{+}$(see [10] for details).

For any $\vec{y} \in \mathbf{L}$, the collection $P(\vec{y})_{\vec{x}}:=S_{\vec{y}-\vec{x}}$, for $0 \leq \vec{x} \leq \vec{c}$, defines a $\Lambda$-module $P(\vec{y})$, where the maps $P(\vec{y})_{\alpha_{j}^{(i)}}$, are given by the multiplications $X_{i}: S_{\vec{y}-j \vec{x}_{i}} \rightarrow$ $S_{\vec{y}-(j-1) \vec{x}_{i}}$, for $i \in[t]$ and $j \in\left[p_{i}\right]$. It is easy to observe that:

- $P(\vec{y}) \neq 0$ if and only if $\vec{y} \in \mathbf{L}_{+}$,

- $\{P(\vec{y})\}_{0 \leq \vec{y} \leq \vec{c}}$ forms a complete set of nonisomorphic projective $\Lambda$-modules,

- for any $i \in[t]$, the multiplication $X_{i}$. induces the canonical $\Lambda$-homomorphisms $P(\vec{y}) \rightarrow P\left(\vec{y}+\vec{x}_{i}\right)$, for $\vec{y} \in \mathbf{L}_{+}$.

Let $\vec{x}, \vec{y} \in \mathbf{L}_{+}$be a pair such that $\vec{y}=\vec{x}+j \vec{x}_{i}$ for some $(i, j)$ with $0 \leq j \leq p_{i}-1$. Then for any $k$-homomorphism $\psi: V \rightarrow W$, the map $\psi \otimes X_{i}^{j}: V \otimes_{k} P(\vec{x}) \rightarrow W \otimes$ ${ }_{k} P(\vec{y})$ is called a $\Lambda$-homomorphism determined by $\psi$. (Note that $X_{i}^{j}$ is unique, up to scalar, $\Lambda$-homomorphism from $P(\vec{x})$ to $P(\vec{y})$ !). Clearly, this definition can be extended to the matrix context.

Our description of matrix presentations for homogeneous modules is based on the following general result. 
Theorem Let $\Lambda$ be a tubular canonical algebra of type $\mathbf{p}=\left(p_{1}, \ldots, p_{t}\right), N:=N_{\mathbf{p}}$ the $\xi$-matrix from the table

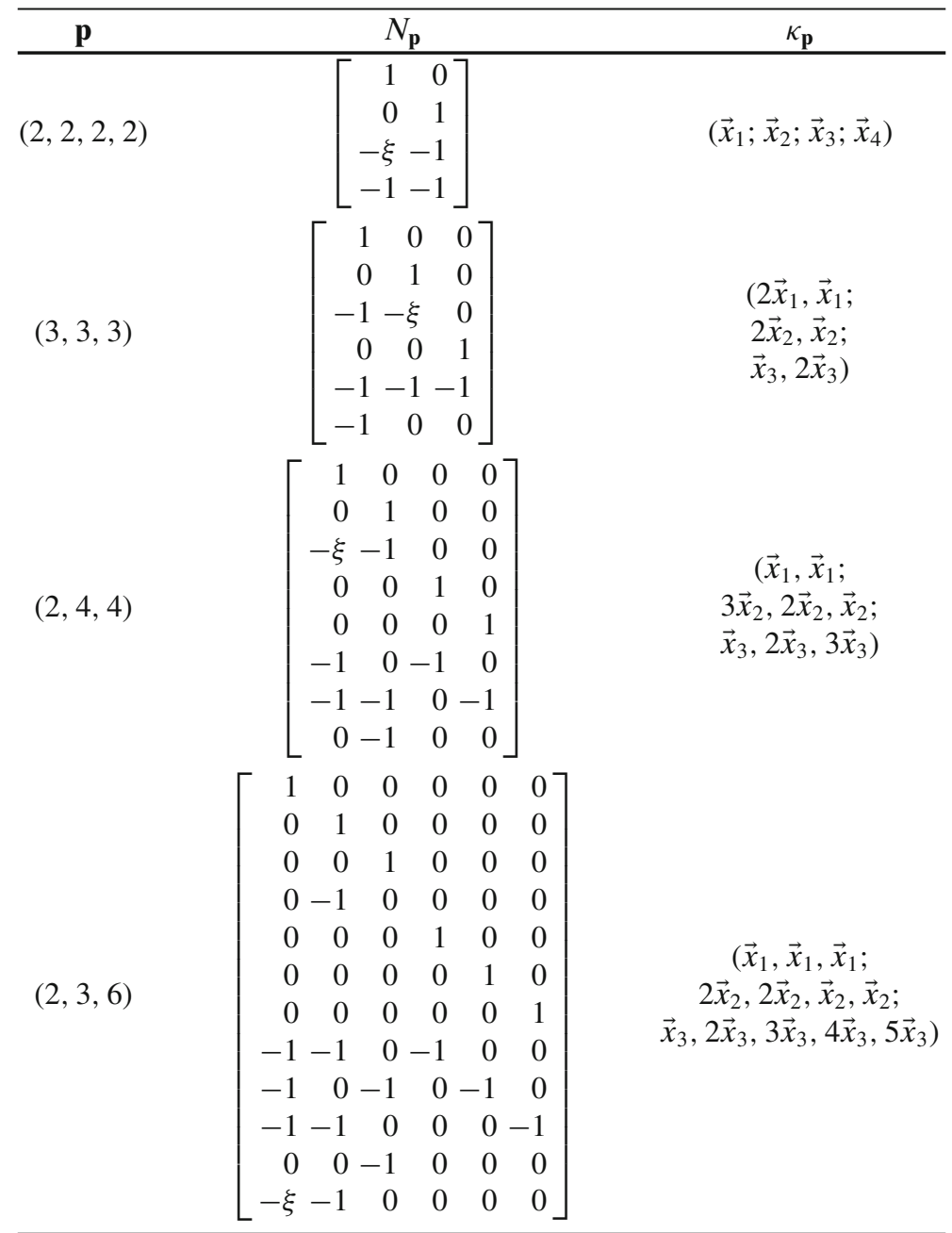

$C^{(n)}$, for any $n \in \mathbb{N}_{\geq p}$, the $k[\xi] \otimes_{k} \Lambda$-module $C^{(n)}:=$ Coker $N^{(n)}$, where

$$
N^{(n)}: \bigoplus_{p} k[\xi] \otimes_{k} P\left((n-p) \vec{x}_{t}\right) \longrightarrow \bigoplus_{i \in[t]} \bigoplus_{j \in\left[p_{i}-1\right]} \bigoplus_{\frac{p}{p_{i}}} k[\xi] \otimes_{k} P\left((n-p) \vec{x}_{t}+j \vec{x}_{i}\right)
$$

is determined by $N_{\mathbf{p}}$, under the ordering of the indices in codomain defined by the sequence $\kappa_{\mathbf{p}}$ as in the table, and

$$
f_{\mathbf{p}}:=\left\{\begin{array}{lll}
\xi(\xi-1)(\xi-\lambda) & \text { if } \quad \mathbf{p}=(2,2,2,2) \\
\xi(\xi-1) & \text { if } \quad \mathbf{p} \neq(2,2,2,2)
\end{array}\right.
$$


Then $C^{(n)}$ belongs to $\bmod _{k[\xi]} \Lambda$ and the $k[\xi]_{f} \otimes_{k} \Lambda$-module $\left(C^{(n)}\right)_{f}$, where $f=f_{\mathbf{p}}$, is a parametrizing bimodule for all indecomposable $\Lambda$-modules from the family of all homogeneous tubes in $\mathcal{X}_{n}$, for every $n \in \mathbb{N}_{\geq p}$.

The proof of Theorem 2.4 needs some preparation and will be given in Section 3.5. We start by formulating an important property of the modules $P(\vec{y})$.

Lemma Let $\Lambda$ be a canonical algebra. Then for any $\vec{x} \in \mathbf{L}_{+}$, the mapping $P(\vec{y}) \mapsto$ $P(\vec{y}+\vec{x})$, for $0 \leq \vec{y} \leq \vec{c}$, extends canonically to functor $\Delta_{\vec{x}}$ between the full subcategories formed by $\{P(\vec{y})\}_{0 \leq \vec{y} \leq \vec{c}}$ and $\{P(\vec{y}+\vec{x})\}_{0 \leq \vec{y} \leq \vec{c}}$.

Proof An easy check on the definition.

The main idea of the proof of Theorem 2.4 relies on an approximation of our parametrizing tensor product functors $-\otimes_{k[\xi]_{f}}\left(C^{(n)}\right)_{f}$ by some well behaved functors associated to $\Delta_{(n-p) \vec{x}_{t}}$, defined on the derived category level. We explain the details in the next section.

\section{The Functors $\tilde{\Delta}_{(n)}$ and $\Delta_{(n)}^{0}$}

For a canonical algebra $\Lambda$ of the type $\mathbf{p}=\left(p_{1}, \ldots, p_{t}\right)$ we denote by $\operatorname{pr} \Lambda$ the category consisting of all finite dimensional projective $\Lambda$-modules and by $\operatorname{pr}_{0} \Lambda$ its full subcategory which is formed by all direct sums of the modules $P(\vec{y})$, for $0 \leq \vec{y} \leq \vec{c}$.

Let $F: \operatorname{pr}_{0} \Lambda \rightarrow \bmod \Gamma$ be an additive $k$-functor, where $\Gamma$ is a $k$-algebra. Then by $\tilde{F}$ and $F^{0}$ we denote the compositions of functors,

$$
\mathcal{K}^{b}\left(\operatorname{pr}_{0} \Lambda\right) \stackrel{\mathcal{K}^{b}(F)}{\longrightarrow} \mathcal{K}^{b}(\bmod \Gamma) \stackrel{Q}{\longrightarrow} \mathcal{D}^{b}(\bmod \Gamma)
$$

and

$$
\bmod \Lambda \stackrel{\mathcal{E}^{0}}{\longrightarrow} \mathcal{D}^{b}(\bmod \Lambda) \stackrel{P^{\bullet}}{\longrightarrow} \mathcal{K}^{b}\left(\operatorname{pr}_{0} \Lambda\right) \stackrel{\tilde{F}}{\longrightarrow} \mathcal{D}^{b}(\bmod \Gamma) \stackrel{H^{0}}{\longrightarrow} \bmod \Gamma
$$

respectively, where $\mathcal{D}^{b}(\bmod \Lambda)\left(\operatorname{resp} . \mathcal{D}^{b}(\bmod \Gamma)\right)$ is the bounded derived category of finite dimensional $\Lambda$ modules (resp. $\Gamma$-modules), $Q$ is canonical quotient functor, $\mathcal{E}^{0}$ is the canonical embedding, $H^{0}$ the "zero homology functor" and $P^{\bullet}$ is a quasiinverse of the canonical functor $\mathcal{K}^{b}(\operatorname{pr} \Lambda) \rightarrow \mathcal{D}^{b}(\bmod \Lambda)$. Recall that generally the functor $P^{\bullet}$ is realized by attaching to any bounded complex $X^{\bullet}$ a fixed complex $P^{\bullet}\left(X^{\bullet}\right)$ in $\mathcal{K}^{-}(\operatorname{pr} \Lambda)$ which is quasi-isomorphic to $X^{\bullet}$, together with a fixed quasiisomorphism $\delta_{X} \bullet: P^{\bullet}\left(X^{\bullet}\right) \rightarrow X^{\bullet}$. In particular, the composition $P^{\bullet} \circ \mathcal{E}^{0}$ attaches to a $\Lambda$-module $M$ its fixed projective resolution. Since $\operatorname{gl} \operatorname{dim} \Lambda<\infty$, we can assume that $P^{\bullet}\left(X^{\bullet}\right)$ belongs to $\mathcal{K}^{b}(\operatorname{pr} \Lambda)$; in fact, even to $\mathcal{K}^{b}\left(\operatorname{pr}_{0} \Lambda\right)$. Note that different choices of complexes $P^{\bullet}\left(X^{\bullet}\right)$, for $X^{\bullet}$ in $\mathcal{D}^{b}(\bmod \Lambda)$, lead to isomorphic functors.

Denote by $\Delta_{(n)}$, for $n \in \mathbb{N}$, the canonical extension of the functor $\Delta_{n \vec{x}_{t}}$ to $\operatorname{pr}_{0} \Lambda$ (here $\Gamma=\Lambda$, see Lemma 2.4 for the definition). If $F=\Delta_{(n)}$, the functors $\tilde{F}=\tilde{\Delta}_{(n)}$ and $F^{0}=\Delta_{(n)}^{0}$ have very nice properties which we discuss below. 
Recall that to any algebra $\Lambda=(\mathbf{p}, \lambda)$ we associate the so-called weighted projective line $\mathbb{X}=\mathbb{X}(\mathbf{p}, \lambda)$ defined by setting $\mathbb{X}:=\operatorname{Proj}^{\mathbf{L}(\mathbf{p})}(S)$ and we consider the abelian hereditary category coh $\mathbb{X}$ consisting of all coherent sheaves of $\mathbf{L}(\mathbf{p})$-graded modules over $\mathbb{X}$. Then the sheaf $\mathcal{T}:=\bigoplus_{0 \leq \vec{x} \leq \vec{c}} \mathcal{O}(\vec{x})$, formed by summing up the all shifted structure sheaves $\mathcal{O}(\vec{x}):=\mathcal{O}_{\mathbb{X}}(\vec{x})$ by elements in $\mathbf{L}(\mathbf{p})$ such that $0 \leq \vec{x} \leq \vec{c}$, is a tilting object in $\operatorname{coh} \mathbb{X}$ and $\operatorname{End}_{\mathbb{X}}(\mathcal{T}) \cong \Lambda$. Moroever, the full right derived functor $\Psi:=$ $\mathbb{R} \operatorname{Hom}_{\mathbb{X}}(\mathcal{T},-): \mathcal{D}^{b}(\operatorname{coh} \mathbb{X}) \rightarrow \mathcal{D}^{b}(\bmod \Lambda)$, for the functor $\operatorname{Hom}_{\mathbb{X}}(\mathcal{T},-): \operatorname{coh} \mathbb{X} \rightarrow$ $\bmod \Lambda$, yields the triangle equivalence $\mathcal{D}^{b}(\operatorname{coh} \mathbb{X}) \simeq \mathcal{D}^{b}(\bmod \Lambda)$ (see [10] for all details).

Let now $\Lambda$ be a tubular canonical algebra of the type p. Then for any $n \in \mathbb{N}_{\geq 1}$ we denote by $\mathcal{X}_{p+n}^{\prime}$ the full subcategory of $\mathcal{X}_{p+n}$ formed by all $\Lambda$-modules nonisomorphic to those lying on the coray ending with the module $\Psi\left(\mathcal{O}\left(\vec{c}+\vec{\omega}+n \vec{x}_{t}\right)\right)$, where $\vec{\omega}:=$ $(t-2) \vec{c}-\sum_{i=1}^{t} \vec{x}_{i} \in \mathbf{L}(\mathbf{p})$ is the so-called dualizing element. (From the general theory it is known that $\Psi\left(\mathcal{O}\left(\vec{c}+\vec{\omega}+n \vec{x}_{t}\right)\right)=\operatorname{Hom}_{\mathbb{X}}\left(\mathcal{T}, \mathcal{O}\left(\vec{c}+\vec{\omega}+n \vec{x}_{t}\right)\right)$ is a $\Lambda$-module lying in the mouth of one of the stable tubes with maximal rank in $\mathcal{X}_{p+n}$, see [10] and also $[8,2.3])$. We set also $\mathcal{X}_{p}^{\prime}:=\mathcal{X}_{p}$.

Theorem Let $\Lambda$ be a tubular canonical algebra of the type $\mathbf{p}=\left(p_{1}, \ldots, p_{t}\right)$. Then for any $n \in \mathbb{N}$, the functor $\tilde{\Delta}_{(n)} \circ P^{\bullet}$ is a triangle autoequivalence of $\mathcal{D}^{b}(\bmod \Lambda)$ which sends $\mathcal{X}_{p}$ into $\mathcal{X}_{p+n}$ and induces the equivalence $\mathcal{X}_{p} \simeq \mathcal{X}_{p+n}^{\prime}$. In particular:

(a) $\left(\tilde{\Delta}_{(n)} \circ P^{\bullet}\right)_{\mid \mathcal{X}_{p}} \cong \Delta_{(n) \mid \mathcal{X}_{p}}^{0}$,

(b) $\Delta_{(n) \mid \mathcal{X}_{p}}^{0}$ yields the equivalence $\mathcal{X}_{p} \simeq \mathcal{X}_{p+n}^{\prime}$.

The proof of Theorem 3.2 needs some preparatory facts. The first one has a rather general character.

Lemma Let $\mathcal{A}$ be an abelian category having enough injective objects, $T$ an object in $\mathcal{A}$ with $E:=\operatorname{End}_{\mathcal{A}}(T)$ and $\mathcal{U}$ the full subcategory of $\mathcal{D}^{+}(\mathcal{A})$ formed by all complexes $X^{\bullet}=\left(X^{i}, d^{i}\right)_{i \in \mathbb{Z}}$ satisfying the following condition:

$$
\operatorname{Ext}_{\mathcal{A}}^{j}\left(T, X^{i}\right)=0 \text {, for all pairs }(i, j) \in \mathbb{Z} \times \mathbb{N}_{\geq 1} \text {. }
$$

Then the mapping $X^{\bullet} \mapsto \operatorname{Hom}_{\mathcal{A}}\left(T, X^{\bullet}\right)$ yields a functor $U=U_{T}: \mathcal{U} \rightarrow$ $\mathcal{D}^{+}(\mathrm{MOD} E)$ and $U \cong \Psi_{\mathcal{U}}$, where $\Psi:=\mathbb{R} \operatorname{Hom}_{\mathcal{A}}(T,-)$ is a full right derived functor of the functor $\operatorname{Hom}_{\mathcal{A}}(T,-): \mathcal{A} \rightarrow \operatorname{MOD} E$

Proof For any $X^{\bullet}$ in $\mathcal{U}$ we fix a quasi-isomorphism $\gamma_{X^{\bullet}}^{\bullet}: X^{\bullet} \rightarrow I^{\bullet}\left(X^{\bullet}\right)$ constructed as in $[11,12]$, where $I^{\bullet}\left(X^{\bullet}\right)$ is a complex bounded below, consisting of injective objects. We show that the map $\operatorname{Hom}_{\mathcal{A}}\left(T, \gamma_{X^{\bullet}}^{\bullet}\right): \operatorname{Hom}_{\mathcal{A}}\left(T, X^{\bullet}\right) \rightarrow \operatorname{Hom}_{\mathcal{A}}\left(T, I^{\bullet}\left(X^{\bullet}\right)\right)$ of complexes of $E$-modules is a quasi-isomorphism, for every $X^{\bullet}$ in $\mathcal{U}$. Observe, that if now $g^{\bullet}: X^{\bullet} \rightarrow Y^{\bullet}$, for $Y^{\bullet}$ in $\mathcal{U}$, is a quasi-isomorphism, then there exists a complex morphism $h^{\bullet}: I^{\bullet}\left(X^{\bullet}\right) \rightarrow I^{\bullet}\left(Y^{\bullet}\right)$ such that $h^{\bullet} \circ \gamma_{X^{\bullet}}^{\bullet}=\gamma_{Y^{\bullet}}^{\bullet} g^{\bullet}\left(h^{\bullet}\right.$ is determined uniquely up to homotopy by $\left.g^{\bullet}\right)$. Hence, $h^{\bullet}$ is a quasi-isomorphism, so it is invertible in the homotopy category. Consequently, so is $\operatorname{Hom}_{\mathcal{A}}\left(T, h^{\bullet}\right)$; hence, 
$\operatorname{Hom}_{\mathcal{A}}\left(T, g^{\bullet}\right)$ is a quasi-isomorphism, since so are the remaining three maps in the equality $\operatorname{Hom}_{\mathcal{A}}\left(T, h^{\bullet}\right) \circ \operatorname{Hom}_{\mathcal{A}}\left(T, \gamma_{X^{\bullet}}^{\bullet}\right)=\operatorname{Hom}_{\mathcal{A}}\left(T, \gamma_{Y^{\bullet}}^{\bullet}\right) \circ \operatorname{Hom}_{\mathcal{A}}\left(T, g^{\bullet}\right)$. Thus $U: \mathcal{U} \rightarrow \mathcal{D}^{+}(\operatorname{MOD} E)$ is really well defined and the family $\left\{\gamma_{X^{\bullet}}\right\}_{X \cdot \in \mathcal{U}}$ of quasiisomorphisms yields the isomorphism $U \cong \Psi_{\mid \mathcal{U}}$. (Recall that we can assume that $\Psi_{\mid \mathcal{U}}$ is given by the formula $\left.\Psi\left(X^{\bullet}\right)=\operatorname{Hom}_{\mathcal{A}}\left(T, I^{\bullet}\left(X^{\bullet}\right)\right)\right)$.

To prove our claim we show first that if $X^{\bullet}=\left(X^{i}, d^{i}\right)_{i \in \mathbb{Z}}$ satisfies $(*)$ and $X^{\bullet}$ is acyclic then the complex $\operatorname{Hom}_{\mathcal{A}}\left(T, X^{\bullet}\right)$ is also acyclic. Without loss of generality we can assume that $X^{i}=0$, for $i<0$.

Set $K^{i}:=\operatorname{Ker} d^{i}$, for any $i \in \mathbb{Z}$. Then we have the exact sequences

$$
\eta_{i}: 0 \rightarrow K^{i} \stackrel{\varepsilon^{i}}{\longrightarrow} X^{i} \stackrel{\pi^{i}}{\longrightarrow} K^{i+1} \rightarrow 0
$$

where $\varepsilon^{i}: K^{i} \rightarrow X^{i}$ and $\pi^{i}: X^{i} \rightarrow K^{i+1}$ are standard embeddings and projections such that $\varepsilon^{i+1} \pi^{i}=d^{i}$. Note that $\operatorname{Ext}_{\mathcal{A}}^{1}\left(T, K^{i}\right)=0$, for every $i \in \mathbb{Z}$, since $K^{1}=X^{0}$ and by $(*)$, from the long exact sequences for $\eta_{i}$ 's we have $\operatorname{Ext}_{\mathcal{A}}^{j}\left(T, K^{i+1}\right)=$ $\operatorname{Ext}_{\mathcal{A}}^{j+1}\left(T, K^{i}\right)$, for all $(i, j) \in \mathbb{Z} \times \mathbb{N}_{\geq 1}$. Then all the sequences $\operatorname{Hom}_{\mathcal{A}}\left(T, \eta_{i}\right)$ are exact in MOD $E$. Hence, the inclusions $\operatorname{Im}_{\operatorname{Hom}}\left(T, d^{i-1}\right) \subseteq \operatorname{Im}_{\operatorname{Hom}_{\mathcal{A}}}\left(T, \varepsilon^{i}\right)$ are in fact the equalities, since $\operatorname{Hom}_{\mathcal{A}}\left(T, \pi^{i-1}\right)$ is surjective for every $i$. On other hand the maps $\operatorname{Hom}_{\mathcal{A}}\left(T, \varepsilon^{i}\right)$ induce the isomorphisms $\operatorname{Ker}_{\operatorname{Hom}}\left(T, d^{i}\right) \cong \operatorname{Hom}_{\mathcal{A}}\left(T, K^{i}\right)$ Consequently, $\quad H^{i}\left(\operatorname{Hom}_{\mathcal{A}}\left(T, X^{\bullet}\right)\right)=\left(\operatorname{Ker}_{\operatorname{Hom}}\left(T, d^{i}\right)\right) /\left(\operatorname{Im}_{\operatorname{Hom}_{\mathcal{A}}}\left(T, d^{i-1}\right)\right)=0$ for every $i \in \mathbb{Z}$, and the complex $\operatorname{Hom}_{\mathcal{A}}\left(T, X^{\bullet}\right)$ is acyclic.

Now fix $X^{\bullet}$ in $\mathcal{U}$ and denote by $Z^{\bullet}$ the (bounded below) complex, formed by the cokernels $Z^{i}:=\operatorname{Coker} \gamma_{X^{\bullet}}^{i}$, for $i \in \mathbb{Z}$. By construction, $\gamma_{X^{\bullet}}^{\bullet}$ is a monomorphism, so the sequence

$$
\eta: \quad 0 \rightarrow X^{\bullet} \stackrel{\gamma_{X}^{\bullet}}{\longrightarrow} I^{\bullet}\left(X^{\bullet}\right) \rightarrow Z^{\bullet} \rightarrow 0
$$

of complexes is exact. Hence, the complex $Z^{\bullet}$ is acyclic, since $\gamma_{X^{\bullet}}^{\bullet}$ is a quasiisomorphism. Moreover, due to property $(*)$ for $X^{\bullet}$, the sequence

$$
\operatorname{Hom}_{\mathcal{A}}(T, \eta): \quad 0 \rightarrow \operatorname{Hom}_{\mathcal{A}}\left(T, X^{\bullet}\right) \stackrel{\ddot{\gamma}_{X}^{\bullet}}{\longrightarrow} \operatorname{Hom}_{\mathcal{A}}\left(T, I^{\bullet}\left(X^{\bullet}\right)\right) \rightarrow \operatorname{Hom}_{\mathcal{A}}\left(T, Z^{\bullet}\right) \rightarrow 0
$$

where $\ddot{\gamma}_{X^{\bullet}}^{\bullet}=\operatorname{Hom}_{\mathcal{A}}\left(T, \gamma_{X^{\bullet}}^{\bullet}\right)$, is also exact and $\operatorname{Ext}_{\mathcal{A}}^{j}\left(T, Z^{i}\right)=0$, for all $(i, j) \in \mathbb{Z} \times \mathbb{N}_{\geq 1}$. Consequently, by the first part of the proof, $\operatorname{Hom}_{\mathcal{A}}\left(T, Z^{\bullet}\right)$ is acyclic. Thus, $\operatorname{Hom}_{\mathcal{A}}\left(T, \gamma_{X^{\bullet}}^{\bullet}\right)$ is a quasi-isomorphism and the proof is complete.

To apply the lemma above in the proof of Theorem 3.2 we need some information on the vanishing of extension groups between shifted structure sheaves $\mathcal{O}(\vec{x})$, for $\vec{x} \in \mathbf{L}=\mathbf{L}(\mathbf{p})$, for arbitrary fixed weighted projective line $\mathbb{X}=\mathbb{X}(\mathbf{p}, \lambda)$ associated to the canonical algebra $\Lambda=\Lambda(\mathbf{p}, \lambda)$.

Lemma $\operatorname{Ext}_{\mathbb{X}}^{1}\left(\mathcal{O}(\vec{x}), \mathcal{O}\left(\vec{y}+n \vec{x}_{t}\right)\right)=0$ for all $0 \leq \vec{x}, \vec{y} \leq \vec{c}$ and $n \in \mathbb{N}$.

Proof We use in the proof the Serre duality formula (see [10]). It states that for any $\mathcal{F}, \mathcal{G}$ in $\operatorname{coh} \mathbb{X}$, the $k$-isomorphism

$$
\operatorname{Ext}_{\mathbb{X}}^{1}(\mathcal{F}, \mathcal{G}) \cong D \operatorname{Hom}_{\mathbb{X}}(\mathcal{G}, \mathcal{F}(\vec{\omega}))
$$

holds, where $\mathcal{F}(\vec{\omega})$ denotes the shift of $\mathcal{F}$ by the dualizing element $\vec{\omega}$. 
Fix $\vec{x}, \vec{y}$ and $n$ as above. Then we have

$$
\begin{aligned}
\operatorname{Ext}_{\mathbb{X}}^{1}\left(\mathcal{O}(\vec{x}), \mathcal{O}\left(\vec{y}+n \vec{x}_{t}\right)\right) & \cong \operatorname{DHom}_{\mathbb{X}}\left(\mathcal{O}\left(\vec{y}+n \vec{x}_{t}\right), \mathcal{O}(\vec{x}+\vec{\omega})\right) \\
& \cong D \operatorname{Hom}_{\mathbb{X}}\left(\mathcal{O}, \mathcal{O}\left(\vec{x}+\vec{\omega}-\vec{y}-n \vec{x}_{t}\right)\right)
\end{aligned}
$$

It is well known that $\operatorname{Hom}_{\mathbb{X}}(\mathcal{O}, \mathcal{O}(\vec{z}))=S_{\vec{z}}$, for any $\vec{z} \in \mathbf{L}$; moreover, that $S_{\vec{z}}=0$ if and only if $\vec{z} \notin \mathbf{L}_{+}$; or equivalently, if $l<0$, where $\vec{z}=l \vec{c}+\sum_{i=1}^{t} l_{i} \vec{x}_{i}$ is the normal form presentation of $\vec{z}$, i.e. $0 \leq l_{i}<p_{i}$ for $i \in[t], l \in \mathbb{Z}$. Note that if $\vec{z}^{\prime}=l^{\prime} \vec{c}+\sum_{i=1}^{t} l_{i}^{\prime} \vec{x}_{i}$ is a normal form presentation of $\vec{z}^{\prime} \in \mathbf{L}$ then $\vec{z} \leq \vec{z}^{\prime}$ if and only if $l^{\prime}-l \geq \mid\left\{i\right.$ : $l_{i}^{\prime}<$ $\left.l_{i}\right\} \mid(\geq 0)$. Therefore it suffices to estimate properly $l$, for $\vec{z}:=\vec{x}+\vec{\omega}-\vec{y}-n \vec{x}_{t}$.

Set $q:=$ quo $_{p_{t}}(n)$ and $r:=\operatorname{rem}_{p_{t}}(n)$. Applying the relations in between the generators in $\mathbf{L}$ and inequalities $\vec{x} \leq \vec{c}, 0 \leq \vec{y}, 0 \leq r \vec{x}_{t}$, we have the following:

$$
\begin{aligned}
\vec{z} & =(t-2) \vec{c}-\sum_{i=1}^{t} \vec{x}_{i}+\vec{x}-\vec{y}-n \vec{x}_{t}=(t-2) \vec{c}-t \vec{c}+\sum_{i=1}^{t}\left(p_{i}-1\right) \vec{x}_{i}+\vec{x}-\vec{y}-n \vec{x}_{t} \\
& \leq-2 \vec{c}+\sum_{i=1}^{t}\left(p_{i}-1\right) \vec{x}_{i}+\vec{x}-n \vec{x}_{t} \leq-\vec{c}+\sum_{i=1}^{t}\left(p_{i}-1\right) \vec{x}_{i}-n \vec{x}_{t} \\
& \leq-(q+1) \vec{c}+\sum_{i=1}^{t}\left(p_{i}-1\right) \vec{x}_{i}
\end{aligned}
$$

Now, from the interpretation of the relation $\leq$ in terms of normal forms as above we obtain $l \leq-(q+1) \leq-1$. Consequently, $\operatorname{Ext}_{\mathbb{X}}^{1}\left(\mathcal{O}(\vec{x}), \mathcal{O}\left(\vec{y}+n \vec{x}_{t}\right)\right)=0$.

Proof of Theorem 3.2 A crucial role in the proof plays the shift functor $\mathcal{O}\left(\vec{x}_{t}\right) \otimes$ $-: \operatorname{coh} \mathbb{X} \rightarrow \operatorname{coh} \mathbb{X}$, induced by an element $\vec{x}_{t} \in \mathbf{L}$. Note that since $\mathcal{O}\left(\vec{x}_{t}\right) \otimes-$ is an exact equivalence, so it induces a triangle equivalence $\mathcal{S}: \mathcal{D}^{b}(\operatorname{coh} \mathbb{X}) \rightarrow \mathcal{D}^{b}(\operatorname{coh} \mathbb{X})$ defined by the formula $\mathcal{S}\left(\mathcal{F}^{\bullet}\right)=\left(\mathcal{O}\left(\vec{x}_{t}\right) \otimes \mathcal{F}^{i}, \mathcal{O}\left(\vec{x}_{t}\right) \otimes d^{i}\right)_{i \in \mathbb{Z}}$, for a bounded complex $\mathcal{F}^{\bullet}=\left(\mathcal{F}^{i}, d^{i}\right)_{i \in \mathbb{Z}}$.

Fix $n \in \mathbb{N}$ and consider the composition

$$
\Psi \circ \mathcal{S}^{n} \circ \Phi: \mathcal{D}^{b}(\bmod \Lambda) \rightarrow \mathcal{D}^{b}(\bmod \Lambda)
$$

where $\Phi:=\mathbb{L}(-\otimes \mathcal{T}): \mathcal{D}^{b}(\bmod \Lambda) \rightarrow \mathcal{D}^{b}(\operatorname{coh} \mathbb{X})$ is a full left derived functor for the tensor product functor $(-\otimes \mathcal{T})$, defined by the tilting object $\mathcal{T}$, and is a quasiinverse triangle equivalence for $\Psi$ (see Section 3.2). We show that $\Psi \circ \mathcal{S}^{n} \circ \Phi \cong$ $\tilde{\Delta}_{(n)} \circ P^{\bullet}$, equivalently, that

$$
\Psi \circ \mathcal{S}^{n} \circ(-\otimes \mathcal{T}) \cong \tilde{\Delta}_{(n)}
$$

as functors from $\mathcal{K}^{b}\left(\operatorname{pr}_{0} \Lambda\right)$ to $\mathcal{D}^{b}(\bmod \Lambda)$. 
Let $X^{\bullet}=\left(X^{i}, d^{i}\right)_{i \in \mathbb{Z}}$ be a complex in $\mathcal{K}^{b}\left(\operatorname{pr}_{0} \Lambda\right)$, where $X^{i}=\bigoplus_{0 \leq \vec{x} \leq \vec{c}} P(\vec{x})^{m_{i, \vec{x}}}$ for any $i$. Then applying the definition of the functors we have

$$
\mathcal{S}^{n}\left(X^{\bullet} \otimes \mathcal{T}\right)=\left(\bigoplus_{0 \leq \vec{x} \leq \vec{c}} \mathcal{O}\left(\vec{x}+n \vec{x}_{t}\right)^{m_{i, \vec{x}}}, \dot{d}^{i}\right)_{i \in \mathbb{Z}}
$$

and consequently, by Lemmas 3.2 and 3.3,

$$
\Psi\left(\mathcal{S}^{n}\left(X^{\bullet} \otimes \mathcal{T}\right)\right) \cong\left(\bigoplus_{0 \leq \vec{x} \leq \vec{c}} \operatorname{Hom}_{\mathbb{X}}\left(\mathcal{T}, \mathcal{O}\left(\vec{x}+n \vec{x}_{t}\right)\right)^{m_{i, \vec{x}}}, \ddot{d}^{i}\right)_{i \in \mathbb{Z}}
$$

where $\dot{d}^{i}$,s and $\ddot{d}^{i}$ s are images of the $d^{i}$ 's by the respective functors. Since we have the natural isomorphism $\operatorname{Hom}_{\mathbb{X}}(\mathcal{T}, \mathcal{O}(\vec{y})) \cong P(\vec{y})$, for any $y \in \mathbf{L}$, we obtain the isomorphism

$$
\Psi\left(\mathcal{S}^{n}\left(X^{\bullet} \otimes \mathcal{T}\right)\right) \cong \tilde{\Delta}_{(n)}\left(X^{\bullet}\right)
$$

(the coincidence of the differentials is obvious). It is clear that this isomorphism is natural with respect to $X^{\bullet}$; thus, $(*)$ is proved.

Now the assertions follow from properties of the functor $\mathcal{S}$ and the pair of functors $(\Phi, \Psi)$. Indeed, for any $n \in \mathbb{N}$, we denote by $\mathcal{C}_{p+n}$ the full subcategory of coh $\mathbb{X}$ consisting of all indecomposable sheaves $\mathcal{F}$ with the slope $\mu_{\mathbb{X}}(\mathcal{F})=n+p$ and by $\mathcal{C}_{p+n}^{\prime}$ its full subcategory formed by all sheaves nonisomorphic to those lying on the coray ending with $\mathcal{O}\left(\vec{c}+\vec{\omega}+n \vec{x}_{t}\right)$. Then, by the general results, it is known that $\mathcal{S}^{n}$ yields the equivalences $\mathcal{C}_{p} \simeq \mathcal{C}_{p+n}$ and $\mathcal{C}_{p}^{\prime} \simeq \mathcal{C}_{p+n}^{\prime}$; moreover, $(\Phi, \Psi)$ induces the equivalences $\mathcal{C}_{p}^{\prime} \simeq \mathcal{X}_{p}$ and $\mathcal{C}_{p+n}^{\prime} \simeq \mathcal{X}_{p+n}^{\prime}$, for $n>0$ (see [10, 16], cf. Section 2.1). In this way the proof is complete.

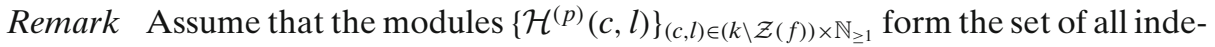
composable nonisomorphic homogeneous $\Lambda$-modules in $\mathcal{X}_{p}$, where $f \in k[\xi]$ and $l$ is a

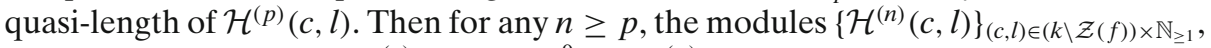
defined by the formula $\mathcal{H}^{(n)}(c, l):=\Delta_{(n-p)}^{0}\left(\mathcal{H}^{(p)}(c, l)\right)$, form the set of all indecomposable nonisomorphic homogeneous $\Lambda$-modules in $\mathcal{X}_{n}$. (We say then that $\mathcal{H}^{(n)}(c, l)$ is a homogeneous module of slope $n$ and quasi-length $l$, "over the point $c$ ").

\section{5}

Proof of Theorem 2.4 We apply Theorem 3.2. Therefore, for each canonical tubular algebra $\Lambda$ of the type $\mathbf{p}$, we give a precise description of the functor $\Delta_{(\bar{n})}^{0}: \bmod \Lambda \rightarrow$ $\bmod \Lambda$ restricted to some subcategory of add $\mathcal{X}_{p}$ containing all homogeneous modules, where $\bar{n}:=n-p$, for $n \in \mathbb{N}_{\geq p}$. For this aim we construct the projective resolutions for this class of $\Lambda$-modules. In particular, we do it for the set of all 
homogeneous $\Lambda$-modules in $\mathcal{X}_{p}$, which in fact are indecomposable homogeneous modules over hereditary algebra $\Lambda_{0}$ of the Euclidean type $\widetilde{\mathbb{D}}_{4}, \widetilde{\mathbb{E}}_{6}, \widetilde{\mathbb{E}}_{7}$ and $\widetilde{\mathbb{E}}_{8}$, respectively. (We can identify $\bmod \Lambda_{0}$ with the full subcategory of $\bmod \Lambda$ formed by all $M=\left(\left(M_{\vec{x}}\right)_{0 \leq \vec{x} \leq \vec{c}},\left(M_{\alpha_{j}^{(i)}}\right)_{i \in[t] ; j \in\left[p_{i}\right]}\right)$ such that $\left.M_{\vec{c}}=0\right)$.

We use the full and faithful functors $\Theta=\Theta_{\mathbf{p}}: \bmod k[\xi] \rightarrow \bmod \Lambda_{0}$, which are based on the "functors $T$ " given in the point 8 of the tables in [3, Section 6] and yield parametrizations of indecomposable homogeneous $\Lambda_{0}$-modules in the respective cases. Below we list the values of $\Theta(V):=\left(\left(M_{\vec{x}}\right)_{0 \leq \vec{x}<\vec{c}},\left(M_{\alpha_{j}^{(i)}}\right)_{i \in[t] ; j \in\left[p_{i}-1\right]}\right)$, for $V \in \bmod k[\xi]$ interpreted as a pair $(V, \varphi)$ consisting of a $k$-space $V$ and a $k$ endomorphism $\varphi$.

$$
\begin{aligned}
\mathbf{p}= & (2,2,2,2): \\
& \left(M_{0} ; M_{\vec{x}_{1}} ; M_{\vec{x}_{2}} ; M_{\vec{x}_{3}} ; M_{\vec{x}_{4}}\right)=\left(V^{2} ; V ; V ; V ; V\right), \\
& \left(M_{\alpha_{1}^{(1)}} ; M_{\alpha_{1}^{(2)}} ; M_{\alpha_{1}^{(3)}} ; M_{\alpha_{1}^{(4)}}\right)=\left(\left[\begin{array}{c}
\mathrm{id} \\
\varphi
\end{array}\right] ;\left[\begin{array}{c}
\mathrm{id} \\
\mathrm{id}
\end{array}\right] ;\left[\begin{array}{c}
0 \\
\mathrm{id}
\end{array}\right] ;\left[\begin{array}{c}
\mathrm{id} \\
0
\end{array}\right]\right) ;
\end{aligned}
$$

$\mathbf{p}=(3,3,3):$

$\left(M_{0} ; M_{\vec{x}_{1}}, M_{2 \vec{x}_{1}} ; M_{\vec{x}_{2}}, M_{2 \vec{x}_{2}} ; M_{\vec{x}_{3}}, M_{2 \vec{x}_{3}}\right)=\left(V^{3} ; V^{2}, V ; V^{2}, V ; V^{2}, V\right)$,

$\left(M_{\alpha_{1}^{(1)}}, M_{\alpha_{2}^{(1)}} ; M_{\alpha_{1}^{(2)}}, M_{\alpha_{2}^{(2)}} ; M_{\alpha_{1}^{(3)}}, M_{\alpha_{2}^{(3)}}\right)$

$$
=\left(\left[\begin{array}{cc}
\mathrm{id} & \varphi \\
\mathrm{id} & \mathrm{id} \\
\mathrm{id} & 0
\end{array}\right],\left[\begin{array}{c}
\mathrm{id} \\
0
\end{array}\right] ;\left[\begin{array}{cc}
\mathrm{id} & 0 \\
0 & \mathrm{id} \\
0 & 0
\end{array}\right],\left[\begin{array}{c}
\mathrm{id} \\
0
\end{array}\right] ;\left[\begin{array}{cc}
0 & 0 \\
\mathrm{id} & 0 \\
0 & \mathrm{id}
\end{array}\right],\left[\begin{array}{c}
0 \\
\mathrm{id}
\end{array}\right]\right) \text {; }
$$

$\mathbf{p}=(2,4,4):$

$$
\begin{aligned}
& \left(M_{0} ; M_{\vec{x}_{1}} ; M_{\vec{x}_{2}}, M_{2 \vec{x}_{2}} M_{3 \vec{x}_{2}} ; M_{\vec{x}_{3}}, M_{2 \vec{x}_{3}}, M_{3 \vec{x}_{3}}\right)=\left(V^{4} ; V^{2} ; V^{3}, V^{2}, V ; V^{3}, V^{2}, V\right) \\
& =\left(\left[\begin{array}{cc}
\varphi & \text { id } \\
\text { id } & 0 \\
\text { id } & \text { id } \\
0 & \text { id }
\end{array}\right] ;\left[\begin{array}{ccc}
\text { id } & 0 & 0 \\
0 & \text { id } & 0 \\
0 & 0 & \text { id } \\
0 & 0 & 0
\end{array}\right],\left[\begin{array}{cc}
\text { id } & 0 \\
0 & \text { id } \\
0 & 0
\end{array}\right],\left[\begin{array}{c}
\text { id } \\
0
\end{array}\right] ;\right. \\
& \left.\left[\begin{array}{ccc}
0 & 0 & 0 \\
\text { id } & 0 & 0 \\
0 & \text { id } & 0 \\
0 & 0 & \text { id }
\end{array}\right],\left[\begin{array}{cc}
0 & 0 \\
\text { id } & 0 \\
0 & \text { id }
\end{array}\right],\left[\begin{array}{c}
0 \\
\text { id }
\end{array}\right]\right)
\end{aligned}
$$




$$
\begin{aligned}
& \mathbf{p}=(2,3,6): \\
& \left(M_{0} ; M_{\vec{x}_{1}} ; M_{\vec{x}_{2}}, M_{2 \vec{x}_{2}} ; M_{\vec{x}_{3}}, M_{2 \vec{x}_{3}}, M_{3 \vec{x}_{3}}, M_{4 \vec{x}_{3}}, M_{5 \vec{x}_{3}}\right) \\
& =\left(V^{6} ; V^{3} ; V^{4}, V^{2} ; V^{5}, V^{4}, V^{3}, V^{2}, V\right), \\
& \left(M_{\alpha_{1}^{(1)}} ; M_{\alpha_{1}^{(2)}}, M_{\alpha_{2}^{(2)}} ; M_{\alpha_{1}^{(3)}}, M_{\alpha_{2}^{(3)}}, M_{\alpha_{3}^{(3)}}, M_{\alpha_{4}^{(3)}}, M_{\alpha_{5}^{(3)}}\right) \\
& =\left(\left[\begin{array}{ccc}
0 & \text { id } & 0 \\
\text { id } & \text { id } & 0 \\
\text { id } & 0 & \text { id } \\
\text { id } & \text { id } & 0 \\
0 & 0 & \text { id } \\
\varphi & \text { id } & 0
\end{array}\right] ;\left[\begin{array}{cccc}
\text { id } & 0 & 0 & 0 \\
0 & \text { id } & 0 & 0 \\
0 & 0 & \text { id } & 0 \\
0 & 0 & 0 & \text { id } \\
0 & 0 & 0 & 0 \\
0 & 0 & 0 & 0
\end{array}\right],\left[\begin{array}{cc}
\text { id } & 0 \\
0 & \text { id } \\
0 & 0 \\
0 & 0
\end{array}\right] ;\left[\begin{array}{ccccc}
0 & 0 & 0 & 0 & 0 \\
\text { id } & 0 & 0 & 0 & 0 \\
0 & \text { id } & 0 & 0 & 0 \\
0 & 0 & \text { id } & 0 & 0 \\
0 & 0 & 0 & \text { id } & 0 \\
0 & 0 & 0 & 0 & \text { id }
\end{array}\right]\right. \text {, } \\
& \left.\left[\begin{array}{cccc}
0 & 0 & 0 & 0 \\
\text { id } & 0 & 0 & 0 \\
0 & \text { id } & 0 & 0 \\
0 & 0 & \text { id } & 0 \\
0 & 0 & 0 & \text { id }
\end{array}\right],\left[\begin{array}{ccc}
0 & 0 & 0 \\
\text { id } & 0 & 0 \\
0 & \text { id } & 0 \\
0 & 0 & \text { id }
\end{array}\right],\left[\begin{array}{cc}
0 & 0 \\
\text { id } & 0 \\
0 & \text { id }
\end{array}\right],\left[\begin{array}{c}
0 \\
\text { id }
\end{array}\right]\right) \text {. }
\end{aligned}
$$

It is well known that $\operatorname{Im} \Theta \subset$ add $\mathcal{X}_{p}$; moreover, the modules

$$
\mathcal{H}^{(p)}(c, l):=\Theta\left(V_{c, l}\right)
$$

for $(c, l) \in(k \backslash \mathcal{Z}(f)) \times \mathbb{N}_{\geq 1}$ form a full list of nonisomorphic homogeneous $\Lambda$ modules in $\mathcal{X}_{p}$, where $V_{c, l}:=\left(k[\xi] /(\xi-c)^{l}, \bar{\xi} \cdot\right) \cong\left(k^{l}, J_{l}(c)\right)$ and $f:=f_{\mathbf{p}}$ (see [16, Section 5.4(i)], cf. Remark 3.4).

Now, observe that the (nonzero) $\Lambda$-modules $\Theta(V)$ have projective dimension 1 and admit projective resolution of the form

$$
0 \longrightarrow V \otimes_{k} P^{1} \stackrel{g}{\longrightarrow} V \otimes_{k} P^{0} \stackrel{g^{\prime}}{\longrightarrow} \Theta(V) \longrightarrow 0
$$

where $P^{1}:=\bigoplus_{p} P(0)$ and $P^{0}:=\bigoplus_{i \in[t]} \bigoplus_{j \in\left[p_{i}-1\right]} \bigoplus_{\frac{p}{p_{i}}} P\left(j \vec{x}_{i}\right)$ (where the ordering of the direct summands is defined by $\left.\kappa_{\mathbf{p}}\right), g=g(V)$ is determined by the matrix $\bar{N}=$ $\bar{N}_{\mathbf{p}}(V)$ with coefficients in $\operatorname{End}_{k}(V)$, obtained from $N=N_{\mathbf{p}}$ by replacing $\xi$ by $\varphi$ and 1 by $\operatorname{id}_{V}$, and $g^{\prime}=g^{\prime}(V)$ is defined under the identifications $V \otimes_{k} S_{\vec{y}} \cong V \otimes_{k} k \cong V$, for $0 \leq \vec{y}<\vec{c}$, by the components $\left(g_{\vec{x}}^{\prime}\right)_{0 \leq \vec{x}<\vec{c}}$ given as follows

$$
g_{\vec{x}}^{\prime}=\left\{\begin{array}{lll}
\operatorname{id} & \text { if } & \vec{x} \neq 0, \\
{\left[M_{\alpha_{1}^{(1)}}\left|M_{\alpha_{1}^{(2)}}\right| \ldots \mid M_{\alpha_{1}^{(t)}}\right]} & \text { if } & \vec{x}=0 .
\end{array}\right.
$$

An easy check on definitions shows that for every considered $\mathbf{p}, g^{\prime}$ is an epimorphism and $g^{\prime} g=0$. The fact that for $g$ is a monomorphism is also almost straightforward. Now the exactness of the sequence above follows immediately by comparison of the dimension vectors.

For any $V$ in $\bmod k[\xi]$, denote by $\Pi^{\bullet}(V)$ the complex

$$
\cdots \rightarrow 0 \longrightarrow V \otimes_{k} P^{1} \stackrel{g}{\longrightarrow} V \otimes_{k} P^{0} \longrightarrow 0 \rightarrow \ldots
$$


in $\mathcal{K}^{-}(\operatorname{pr} \Lambda)$ such that $\Pi^{0}(V)=V \otimes_{k} P^{0}$. Then for a fixed $n \geq p$, the complex $\tilde{\Delta}_{(\bar{n})}\left(\Pi^{\bullet}(V)\right)$ is naturally isomorphic in $\mathcal{D}^{b}(\bmod \Lambda)$ to the complex

$$
\cdots \rightarrow 0 \longrightarrow V \otimes_{k} \hat{P}^{1} \stackrel{\hat{g}}{\longrightarrow} V \otimes_{k} \hat{P}^{0} \longrightarrow 0 \rightarrow \ldots
$$

where $\hat{P}^{1}=\bigoplus_{p} P\left(\bar{n} \vec{x}_{t}\right), \hat{P}^{0}:=\bigoplus_{i \in[t]} \bigoplus_{j \in\left[p_{i}-1\right]} \bigoplus_{\frac{p}{p_{i}}} P\left(\bar{n} \vec{x}_{t}+j \vec{x}_{i}\right)$ (the ordering of direct summands is given by $\left.\kappa_{\mathbf{p}}\right)$ and $\hat{g}=\hat{g}(V)$ is determined by the matrix $\bar{N}=\bar{N}_{\mathbf{p}}(V)$.

Without loss of generality we can assume that $\left(P^{\bullet}(\Theta(-))\right)=\Pi^{\bullet}(-)$. Hence, we have

$$
\left(\Delta_{(\bar{n})}^{0} \circ \Theta\right)(-)=\left(H^{0} \circ \tilde{\Delta}_{(\bar{n})} \circ \Pi^{\bullet}(-)\right)
$$

as functors from $\bmod k[\xi]$ to $\bmod \Lambda$, and

$$
\left(\Delta_{(\bar{n})}^{0} \circ \Theta\right)(-)=\operatorname{Coker} \hat{g}(-) .
$$

Since the map $V \otimes_{k[\xi]} N^{(n)}$ can be canonically identified with $\hat{g}$, by the right exactness of tensor product we have the isomorphism

$$
\left(\Delta_{(\bar{n})}^{0} \circ \Theta\right)(-)=\operatorname{Coker}\left((-) \otimes_{k[\xi]} N^{(n)}\right) \cong-\otimes_{k[\xi]} C^{(n)}
$$

of functors.

Finally observe that, by Theorem 3.2(a), $\operatorname{Ker} \hat{g}(V)=0$ for all $V$ in $\bmod k[\xi]$. Consequently, for any $c \in k$, the sequence

$$
0 \rightarrow k \otimes_{k} \hat{P}^{1} \stackrel{\hat{g}}{\longrightarrow} k \otimes_{k} \hat{P}^{0} \rightarrow V_{c, 1} \otimes_{k[\xi]} C^{(n)} \rightarrow 0
$$

of $\Lambda$-modules is exact, so the dimension sequence $\left\{\operatorname{dim}_{k} V_{c, 1} \otimes_{k[\xi]} C^{(n)}\right\}_{c \in k}$ is constant. This implies that $C^{(n)}$ belongs to $\bmod _{k[\xi]} \Lambda$. Indeed, since $C^{(n)}$ is a finitely generated $k[\xi]$-module, there exists a decomposition $C^{(n)}=C^{\prime} \oplus C^{\prime \prime}$ into a direct sum of $k[\xi]-$ submodules such that $C^{\prime}$ is free and $C^{\prime \prime}$ is torsion. In case $C^{\prime \prime} \neq 0$, then $\operatorname{ann}_{k[\xi]} C^{\prime \prime} \neq$ $k[\xi]$, so $\mathcal{Z}\left(\operatorname{ann}_{k[\xi]} C^{\prime \prime}\right) \neq \emptyset$. Moreover, we have $V_{c, 1} \otimes_{k[\xi]} C^{\prime \prime} \neq 0$ if and only if $c \in$ $\mathcal{Z}\left(\operatorname{ann}_{k[\xi]} C^{\prime \prime}\right)$. On other hand $\left\{\operatorname{dim}_{k} V_{c, 1} \otimes_{k[\xi]} C^{\prime}\right\}_{c \in k}$ is constant, a contradiction. Thus, $C^{\prime \prime}=0$.

Now the proof is complete, since by Theorem 3.2(b) the indecomposable homogeneous $\Lambda$-modules $\left\{\mathcal{H}^{(n)}(c, l)\right\}_{(c, l) \in(k \backslash \mathcal{Z}(f)) \times \mathbb{N}_{\geq 1}}$ of the shape $\mathcal{H}^{(n)}(c, l)=\Delta_{(\bar{n})}^{0}\left(\mathcal{H}^{(p)}(c, l)\right)$ form the set of all nonisomorphic objects in $\mathcal{X}_{n}$ (see Remark 3.4), and $\mathcal{H}^{(n)}(c, l) \cong$ $V_{c, l} \otimes_{k[\xi]_{f}}\left(C^{(n)}\right)_{f}$ by $(*)$ and $(* *)$.

\section{The Main Result}

\section{1}

Let $\Lambda$ be a canonical algebra, $R$ a commutative $k$-algebra and $B$ a module in $\bmod _{R} \Lambda$. Then the module $B^{R}:=\operatorname{Hom}_{R}(B, R)$ in $\operatorname{MOD} R \otimes_{k} \Lambda^{\text {op }}$, in fact in $\bmod _{R} \Lambda^{\text {op }}$, can be regarded naturally as a module in $\bmod _{R} \Lambda$ (we have the canonical $k$-algebra isomorphism $v: \Lambda \stackrel{\sim}{\rightarrow} \Lambda^{\text {op }}$ induced by the quiver isomorphism $Q \stackrel{\sim}{\rightarrow} Q^{\text {op }}$, given by the mapping $j \vec{x}_{i} \mapsto\left(p_{i}-j\right) \vec{x}_{i}$, for all $i \in[t]$ and $\left.0 \leq j \leq p_{i}\right)$. In particular, the usual duality $\operatorname{Hom}_{k}(-, k): \bmod \Lambda \rightarrow \bmod \Lambda^{\text {op }}$ yields via $v$ the selfduality $D: \bmod \Lambda \rightarrow$ $\bmod \Lambda$. Note that if $B=\left(B_{\alpha_{j}^{(i)}}\right)_{i \in[t], j \in\left[p_{i}\right]}$ belongs to $\bmod _{R}^{\text {mat }} \Lambda$ then $B^{R}$ is canonically 
isomorphic to the matrix module $B^{\mathrm{tr}}:=\left(B_{\alpha_{j}^{(i)}}^{\prime}\right)_{i \in[t], j \in\left[p_{i}\right]}$ in $\bmod _{R}^{\mathrm{mat}} \Lambda$, where $B_{\alpha_{j}^{(i)}}^{\prime}=$ $\left(B_{\alpha_{p_{i}+1-j}^{(i)}}\right)^{\operatorname{tr}}$, for all $i \in[t], j \in\left[p_{i}\right]$.

Theorem Let $\Lambda=\Lambda(\mathbf{p}, \lambda)$ be a tubular canonical algebra of type $\mathbf{p}=\left(p_{1}, \ldots, p_{t}\right)$ and $\left\{B^{(n)}\right\}_{n \in \mathbb{Z}_{\geq p}}$ the family of matrix modules from $\bmod _{k[\xi]}^{\operatorname{mat}} \Lambda$ as in Section 4.7. Then for any $n \in \mathbb{Z}_{\geq p} \quad\left(\right.$ resp. $\left.n \in \mathbb{Z}_{\leq 0}\right)$ the matrix module $\left(B^{(n)}\right)_{f} \quad\left(\operatorname{resp} .\left(\left(B^{(p-n)}\right)^{\operatorname{tr}}\right)_{f}\right)$ in $\bmod _{k[\xi]_{f}}^{\text {mat }} \Lambda$, where $f=f_{\mathbf{p}}$, is a parametrizing bimodule for all indecomposable $\Lambda$ modules from the family of all homogeneous tubes in $\mathcal{X}_{n}$.

\section{Remark}

(a) For any pair $(c, l) \in\left(k \backslash \mathcal{Z}\left(f_{\mathbf{p}}\right)\right) \times \mathbb{N}_{\geq 1}$, the matrix module representing the indecomposable homogeneous module $\mathcal{H}^{(n)}(c, l) \cong k[\xi] /(\xi-c)^{l} \otimes_{k[\xi]} B^{(n)}$ of slope $n$ and quasi-length $l$, over the point $c \in k$, can be obtained by replacing in each matrix forming $B^{(n)}$, the scalars $a=0,1, \lambda$ by the matrices $a \cdot I_{l}$ and $\xi$ by $J_{l}(c)$.

(b) The bimodule parametrizing the indecomposable modules from the homogeneous tubes in $\mathcal{X}_{\infty}$ can be easily constructed from that for the case of the canonical hereditary algebra of the type $\tilde{\mathbb{A}}_{p_{1}, p_{2}}$ (see [8]).

To prove Theorem 4.1 we show first how to reduce the case " $n \in \mathbb{Z}_{\leq 0}$ " to the case " $n \in \mathbb{Z}_{\geq p}$ ". The proof of the main part is quite technical. It is presented in Section 4.6.

\section{2}

Assume that $\Lambda=\Lambda(\mathbf{p}, \lambda)$ is as in Theorem 4.1.

Lemma For any $q \in \mathbb{Q}_{\leq 0} \cup \mathbb{Q}_{\geq p}$, the selfduality $D: \bmod \Lambda \rightarrow \bmod \Lambda$ yields an equivalence $\mathcal{X}_{q} \simeq\left(\mathcal{X}_{p-q}\right)^{\mathrm{op}}$.

Proof It suffices to show that for any $\Lambda$-module $M=\left(\left(M_{\vec{x}}\right)_{0 \leq \vec{x} \leq \vec{c}},\left(M_{\alpha_{j}^{(i)}}\right)_{i \in[t] ; j \in\left[p_{i}\right]}\right)$ in $\bmod \Lambda$ with $\mu(M)=q$, the equality $\mu(D(M))=p-q$ holds.

Note that $D(M)=\left(\left(M_{\vec{x}}^{\prime}\right)_{0 \leq \vec{x} \leq \vec{c}},\left(M_{\alpha_{j}^{(i)}}^{\prime}\right)_{i \in[t] ; j \in\left[p_{i}\right]}\right)$, where $M_{j \vec{x}_{i}}^{\prime}=M_{\left(p_{i}-j\right) \vec{x}_{i}}^{*}$ for all $i \in[t]$ and $0 \leq j \leq p_{i}$. Hence, by the definitions we have $\operatorname{rk}(D(M))=-\operatorname{rk}(M)$, $\operatorname{deg}(D(M))=\operatorname{deg}(M)-p \cdot \operatorname{rk}(M)$ and consequently, $\mu(D(M))=p-q$.

Proposition For any $q \in \mathbb{Q}_{\leq 0} \cup \mathbb{Q}_{\geq p}$, if the module $B$, where $B$ is in $\bmod _{k[\xi]} \Lambda$, is a parametrizing bimodule for the whole family of homogeneous tubes in $\mathcal{X}_{q}$ then the module $\left(B_{f}\right)^{k[\xi]_{f}}$ in $\bmod _{k[\xi]_{f}} \Lambda$ is a parametrizing bimodule for the whole family of homogeneous tubes in $\mathcal{X}_{p-q}$.

Proof We start by invoking a certain general fact: Let $R$ be a commutative $k$-algebra, $C$ be a module in $\bmod _{R} \Lambda$ and $N$ an $R$-module. Then we have the following natural left $\Lambda$-module isomorphism:

$$
\operatorname{Hom}_{k}(N, k) \otimes_{R} \operatorname{Hom}_{R}(C, R) \cong \operatorname{Hom}_{R}\left(C, \operatorname{Hom}_{k}(N, k)\right) \cong \operatorname{Hom}_{k}\left(N \otimes_{R} C, k\right)
$$


(the first isomorphism holds since $C$ is $\operatorname{in} \bmod _{R} \Lambda$; the second one is the standard adjunction formula).

Now, applying the isomorphism above to $R:=k[\xi]_{f}$ and $C:=B_{f}$, we obtain via $v: \Lambda \stackrel{\sim}{\rightarrow} \Lambda^{\text {op }}$ an isomorphism $N^{*} \otimes_{k[\xi]_{f}}\left(B_{f}\right)^{k[\xi]_{f}} \cong D\left(N \otimes_{k[\xi]_{f}} B_{f}\right)$ of right $\Lambda$ modules. Observe that for any indecomposable $N$ we have $N^{*} \cong N$, since $c$ is the unique eigenvalue of $J_{l}(c)^{\mathrm{tr}}$, where $N=\left(k^{l}, J_{l}(c)\right)$, for some $(c, l) \in(k \backslash \mathcal{Z}(f)) \times$ $\mathbb{N}_{\geq 1}$. Consequently, for any indecomposable $k[\xi]_{f}$-module $N$ we have the isomorphism $N \otimes_{k[\xi]_{f}}\left(B_{f}\right)^{k[\xi]_{f}} \cong D\left(N \otimes_{k[\xi]_{f}} B_{f}\right)$.

Now the assertion follows immediately from the lemma and the fact that the equivalence $\mathcal{X}_{q} \simeq\left(\mathcal{X}_{p-q}\right)^{\text {op }}$ preserves the tubular structure.

Corollary If under the assumptions of the proposition $B$ belongs to $\bmod _{k[\xi]}^{\operatorname{mat}} \Lambda$ then $\left(B^{\operatorname{tr}}\right)_{f}$ is a parametrizing bimodule for the whole family of homogeneous tubes in $\mathcal{X}_{p-q}$.

Proof Follows immediately from the proposition, by the canonical isomorphisms $\left(B_{f}\right)^{k[\xi]_{f}} \cong\left(B^{k[\xi]}\right)_{f} \cong\left(B^{\operatorname{tr}}\right)_{f}$ in $\bmod _{k[\xi]_{f}} \Lambda$.

\section{3}

To prove the case " $n \in \mathbb{Z}_{\geq p}$ ", we apply for the bimodules $C^{(n)}$ from Theorem 2.4 , a quite general method of determining matrix forms of cokernels.

We say that a matrix $D \in \mathbb{M}_{a \times b}(k[\xi])$ is quasi-diagonal if in each row and column of $D$ there exists at most one nonzero element; the set of all nonzero coefficients is called then a quasi-diagonal of $D$. Clearly, if $D$ is diagonal; in particular, if $D$ has a diagonal normal form in the sense of Smith (see [20]), then $D$ is quasi-diagonal. Consequently, for any matrix $F \in \mathbb{M}_{a \times b}(k[\xi])$ there exist invertible matrices $U \in$ $\mathrm{u}\left(\mathbb{M}_{a}(k[\xi])\right)$ and $V \in \mathrm{u}\left(\mathbb{M}_{b}(k[\xi])\right)$ such that $D:=U F V$ is quasi-diagonal. Any such $D$ we call a quasi-diagonal form of $F$.

Proposition Let $\Lambda=k Q / I$ be an algebra given by a bound quiver $(Q, I)$, where $Q=\left(Q_{0}, Q_{1}\right)$. Moreover, let $E=\left(E_{\alpha}\right)$ and $E^{\prime}=\left(E_{\alpha}^{\prime}\right)$ be a pair of modules in $\bmod _{k[\xi]}^{\text {mat }} \Lambda$ with rank vectors $\underline{\mathrm{rk}}(E)=\left(e_{v}\right), \underline{\mathrm{rk}}\left(E^{\prime}\right)=\left(e_{v}^{\prime}\right) \in \mathbb{N}^{Q_{0}}$, respectively, and $F=$ $\left(F_{v}\right): E^{\prime} \rightarrow E$ a morphism in $\bmod k[\xi] \otimes_{k} \Lambda$, where $F_{v} \in \mathbb{M}_{e_{v} \times e_{v}^{\prime}}(k[\xi])$, for $v \in Q_{0}$. Assume that for any $v \in Q_{0}$, the matrix $D_{v}=U_{v} F_{v} V_{v}$ is a quasi-diagonal form of $F_{v}$, having nonzero elements exactly in rows with increasing indices $\iota_{1}^{v}, \ldots, \iota_{g_{v}}^{v}$, where $U_{v} \in \mathrm{u}\left(\mathbb{M}_{e_{v}}(k[\xi])\right), V_{v} \in \mathrm{u}\left(\mathbb{M}_{e_{v}^{\prime}}(k[\xi])\right)$ and $g_{v} \leq e_{v}, e_{v}^{\prime}$. If the cokernel $C:=$ Coker $F$ belongs to $\bmod _{k[\xi]} \Lambda$ then the all nonzero elements in each of the matrices $D_{v}, v \in Q_{0}$, are invertible and $C$ is isomorphic to the matrix module $\breve{W}=\left(\breve{W}_{\alpha}\right)$ with rank vector $\left(e_{v}-g_{v}\right)_{v \in Q_{0}}$, where each $\breve{W}_{\alpha}$ is the submatrix of the matrix $W_{\alpha}:=$ $U_{s(\alpha)} E_{\alpha} U_{t(\alpha)}^{-1} \in \mathbb{M}_{s(\alpha) \times t(\alpha)}(k[\xi])$ with coordinates in the $\operatorname{set}\left(\left[e_{s(\alpha)}\right] \backslash\left\{\iota_{1}^{s(\alpha)}, \ldots, l_{g_{s(\alpha)}}^{s(\alpha)}\right\}\right) \times$ $\left(\left[e_{t(\alpha)}\right] \backslash\left\{\iota_{1}^{t(\alpha)}, \ldots, \iota_{g_{t(\alpha)}(\alpha)}^{(\alpha)}\right\}\right)$.

Proof Follows immediately if we interpret $\left\{F_{v}\right\}$ and $\left\{E_{\alpha}\right\}$ as maps between the respective free modules with the bases formed by columns of the matrices $\left\{V_{v}\right\}$ and $\left\{U_{v}^{-1}\right\}$. 
Let now $\Lambda=\Lambda(\mathbf{p}, \lambda)$ be a canonical tubular $k$-algebra of type $\mathbf{p}$. To determine for a fixed $n \geq p$, a matrix presentation of the bimodule $C^{(n)}=\operatorname{Coker} N^{(n)}$ we have to proceed in practice as follows:

(P1) for each vertex $v=\vec{x}$, where $0 \leq \vec{x} \leq \vec{c}$, find $k[\xi]$-bases $\mathcal{B}_{v}^{0}=\mathcal{B}_{v}^{0}(n)$ and $\mathcal{B}_{v}^{1}=$ $\mathcal{B}_{v}^{1}(n)$ of the modules $\left(k[\xi] \otimes_{k} \hat{P}^{0}\right)_{v}$ and $\left(k[\xi] \otimes_{k} \hat{P}^{1}\right)_{v}$, where $\hat{P}^{0}=\hat{P}^{0}(n)$ and $\hat{P}^{1}=\hat{P}^{1}(n)$ are as in Section 3.5,

(P2) for each vertex $v$ as above, construct the matrix $F_{v}=F_{v}^{(n)}$ of the map $N_{v}^{(n)}$ with respect to the bases $\mathcal{B}_{v}^{0}$ and $\mathcal{B}_{v}^{1}$, and next find an invertible $\xi$-matrix $U_{v}=U_{v}^{(n)}$ and quasi-diagonal form $D_{v}=D_{v}^{(n)}$ of $F_{v}$ of the shape $D_{v}=U_{v} F_{v} V_{v}$, where $V_{v}=V^{(n)}$ is an invertible $\xi$-matrix,

(P3) for each arrow $\alpha=\alpha_{j}^{(i)}$, construct the matrix $E_{\alpha}=E_{\alpha}^{(n)}$ of the structure map $\left(k[\xi] \otimes_{k} \hat{P}^{0}(n)\right)_{\alpha}$ with respect to the bases $\mathcal{B}_{t(\alpha)}^{0}$ and $\mathcal{B}_{s(\alpha)}^{0}$ and compute the matrix $W_{\alpha}\left(=W_{\alpha}^{(n)}\right)=U_{s(\alpha)} E_{\alpha} U_{t(\alpha)}^{-1}$,

(P4) for each arrow $\alpha=\alpha_{j}^{(i)}$, form the submatrix $\breve{W}_{\alpha}=\breve{W}_{\alpha}^{(n)}$ of $W_{\alpha}$ "determined by the row indices of the quasi-diagonals" in $D_{s(\alpha)}$ and $D_{t(\alpha)}$.

Observe that once we have realized (P1), we can always effectively execute all computations from (P2)-(P4), and obtain a matrix presentation $\bar{C}^{(n)}$ of $C^{(n)}$, which however do not need necessarily coincide with $B^{(n)}$ from Section 4.7. (In particular, in the step (P2) there is no uniqueness!). To prove the theorem we provide a quite restrictive and independent on $n$ way of realization of the program outlined above, showing how to obtain the formula from Section 4.7. It is based on a certain coherent universal choice of the bases and on some rather simple formulas for determining matrices $D_{v}^{(n)}, U_{v}^{(n)}$ and $W_{\alpha}^{(n)}$. These formulas are result of a very specific shape of the matrices $N_{v}^{(n)}$, and reduce the required computations "for all $n$ " to a finite number of cases (reasonable amount, however still to large to present in full version in the article). In particular, they explain the appearance of formulas and series dependent of the reminders modulo $p$.

For any $n \in \mathbb{N}$, we denote by $X=X_{n}$ and $Y=Y_{n}$ the matrices

$$
X=\left[\begin{array}{ccc}
1 & & \\
& \ddots & \\
& & 1 \\
0 & \cdots & 0
\end{array}\right], \quad Y=\left[\begin{array}{ccc}
0 & \cdots & 0 \\
1 & & \\
& \ddots & \\
& & 1
\end{array}\right]
$$

in $\mathbb{M}_{(n+1) \times n}(k)$. Moreover, we set $Z^{\lambda}=Z_{n}^{\lambda}:=X_{n}+\lambda Y_{n}$, for $\lambda \in k$, and $I=I_{n}$, for simplicity we write $Z=Z_{n}^{1}$. Note that we also consider the empty matrices (for $n=0)$.

The following fact plays a fundamental role in our proof and is applied for realization of $(\mathrm{P} 1)$ and first parts of $(\mathrm{P} 2)$ and $(\mathrm{P} 3)$. 
Lemma Let $\Lambda=\Lambda(\mathbf{p}, \lambda)$ be a canonical $k$-algebra.

(a) For any $\vec{y} \in \mathbf{L}_{+}$, the set $\mathcal{B}_{\vec{y}}$ of cosets of the monomials $\left(X_{1}^{p_{1}}\right)^{l-s}\left(X_{2}^{p_{2}}\right)^{s} X_{1}^{l_{1}} \ldots$ $X_{t}^{l_{t}}$, for $s=0, \ldots, l$, where $\vec{y}=l \vec{c}+\sum_{i=1}^{t} l_{i} \vec{x}_{i}$ is the normal form presentation of $\vec{y}$, is a basis of $S_{\vec{y}}$; in particular, $\operatorname{dim}_{k} S_{\vec{y}}=l+1$.

(b) For $\vec{y} \in \mathbf{L}_{+}, i \in[t]$ and $j \in\left[p_{i}-1\right]$, the matrix $\Xi_{\vec{y} ; i, j}$ of the map $X_{i}^{j} \cdot: S_{\vec{y}} \rightarrow S_{\vec{y}^{\prime}}$ in the ordered bases $\mathcal{B}_{\vec{y}}$ and $\mathcal{B}_{\vec{y}^{\prime}}$, for $\vec{y}^{\prime}:=\vec{y}+j \vec{x}_{i}$, is given by the formula:

$$
\Xi_{\vec{y} ; i, j}:=\left\{\begin{array}{lll}
X_{l+1} & \text { if } & l^{\prime}>l, i=1, \\
Y_{l+1} & \text { if } \quad l^{\prime}>l, i=2, \\
Z_{l+1}^{\lambda_{i}} & \text { if } \quad l^{\prime}>l, i \neq 1,2, \\
I_{l+1} & \text { if } \quad l^{\prime}=l,
\end{array}\right.
$$

where $\vec{y}=l \vec{c}+\sum_{i=1}^{t} l_{i} \vec{x}_{i}$ and $\vec{y}^{\prime}=l^{\prime} \vec{c}+\sum_{i=1}^{t} l_{i} \vec{x}_{i}$ are the normal form presentations of $\vec{y}$ and $\vec{y}^{\prime}$, respectively.

Proof An easy check on definitions.

Corollary Let $\vec{y}(\vec{n}, \vec{x}):=\vec{n} \vec{x}_{t}-\vec{x}$, where $\bar{n} \in \mathbb{N}$ and $\vec{x} \in \mathbf{L}$. Then

$$
\Xi_{\vec{y}(\bar{n}, \vec{x}) ; i, j}=\left\{\begin{array}{lll}
X_{l+1+m} & \text { if } & l^{\prime}>l, i=1, \\
Y_{l+1+m} & \text { if } & l^{\prime}>l, i=2, \\
Z_{l+1+m}^{\lambda_{i}} & \text { if } & l^{\prime}>l, i \neq 1,2, \\
I_{l+1+m} & \text { if } & l^{\prime}=l,
\end{array}\right.
$$

where $l, l^{\prime}$ are as above for $\vec{y}:=\vec{y}(r, \vec{x})$ and $\vec{y}^{\prime}:=\vec{y}+j \vec{x}_{i}, r:=\operatorname{rem}_{p}(\vec{n})$ and $m:=$ quo $_{p}(\bar{n})$.

The formula above indicates that the character of the reduction and the cases we have to consider in the proof should depend on $r:=\operatorname{rem}_{p}(\bar{n})$.

Now we formulate the results necessary for the realization of $(\mathrm{P} 2)-(\mathrm{P} 4)$ in our situation. We use a very specific shape of the matrices we deal with.

Let $R$ be a $k$-algebra. Then for any matrix $H=\left[h_{u, w}\right] \in \mathbb{M}_{a \times b}(R)$ and block matrix $G=\left[G_{u, w}\right]_{u \in[a], w \in[b]}$ in $\mathbb{M}_{\sigma \times \tau}(k)$ with blocks $G_{u, w} \in \mathbb{M}_{\sigma_{u} \times \tau_{w}}(k)$, where $\sigma:=$ $\sum_{u=1}^{a} \sigma_{u}$ and $\tau:=\sum_{w=1}^{b} \tau_{w}$, we denote by $H * G$ the block matrix $\left[h_{u, w} \cdot G_{u, w}\right]_{u \in[a], w \in[b]}$ in $\mathbb{M}_{\sigma \times \tau}(R)$ with blocks $h_{u, w} \cdot G_{u, w} \in \mathbb{M}_{\sigma_{u} \times \tau_{w}}(R)$.

We say that a matrix $H=\left[h_{u, w}\right] \in \mathbb{M}_{a \times b}(R)$ is reduced, if there exist $0 \leq \rho \leq a, b$ and an increasing sequence $\underline{u}:=\left(u_{1}, \ldots, u_{\rho}\right)$ of indices such that $h_{u_{z}, z}=1$ for all $z \in$ [ $\rho$ ] and for every $0 \leq z \leq \rho$, we have $h_{u, w}=0$, for all pairs $(u, w)$ with $u_{z} \leq u<u_{z+1}$ and $w>z$, where $u_{0}:=1$ and $u_{\rho+1}:=a+1$. Moreover, we say that $H$ is completely reduced if it is reduced and $h_{u_{z}, w}=0$ for all $z \in[\rho]$ and $w<z$.

Lemma Let $R=k[\xi]$ and let $H=\left[h_{u, w}\right] \in \mathbb{M}_{a \times b}(R)$ be a reduced matrix, $G=\left[G_{u, w}\right]_{u \in[a], w \in[b]}$ in $\mathbb{M}_{\sigma \times \tau}(k)$ a block matrix as above and $D=D^{(H * G)}=$ $\left[D_{u, w}\right]_{u \in[a], w \in[b]}$ a block matrix in $\mathbb{M}_{\sigma \times \tau}(R)$ with the blocks $D_{u, w} \in \mathbb{M}_{\sigma_{u} \times \tau_{w}}(R)$ given 
by $D_{u_{z}, z}:=h_{u_{z}, z} \cdot G_{u_{z}, z}=G_{u_{z}, z}$, for $z \in[\rho]$, and $D_{u, w}:=0$, for the remaining pairs $(u, w)$. Assume that $\sigma_{u_{z}} \geq \tau_{z}$ and $\mathrm{r}\left(G_{u_{z}, z}\right)=\tau_{z}$, for all $z \in[\rho]$. Then:

(a) There exists a block lower unitriangular matrix $\bar{U}=\left[\bar{U}_{u, u^{\prime}}\right]_{u, u^{\prime} \in[a]}$, with $\bar{U}_{u, u^{\prime}} \in$ $\mathbb{M}_{\sigma_{u} \times \sigma_{u^{\prime}}}(R)$ such that $\bar{U}_{u, u^{\prime}}=0$ for $u^{\prime} \neq u_{1}, \ldots, u_{\rho}$ and $u \neq u^{\prime}$, satisfying the equality $\bar{U} \cdot D=H * G$.

(b) If all $G_{u_{z}, z}$, for $z \in[\rho]$, have the form $\left[\begin{array}{c}G(z) \\ 0\end{array}\right]$ (resp. $\left[\begin{array}{c}0 \\ G(z)\end{array}\right]$ ), where $G(z) \in \mathbb{M}_{\tau_{z}}(k)$, then we can set $\bar{U}:=\bar{U}^{(H * G)}$, where the blocks $\bar{U}_{u, u^{\prime}}$, for $u>u^{\prime}$, defining $\bar{U}^{(H * G)}$ are given by the formulas $\bar{U}_{u, u^{\prime}}:=\left[h_{u, z} \cdot\left(G_{u, z} \cdot G(z)^{-1}\right) \mid 0\right]\left(\right.$ resp. $\bar{U}_{u, u^{\prime}}:=$ $\left.\left[0 \mid h_{u, z} \cdot\left(G_{u, z} \cdot G(z)^{-1}\right)\right]\right)$, if $u^{\prime}=u_{z}$ for some $z \in[\rho]$ (and $\bar{U}_{u, u^{\prime}}=0$, otherwise ).

(c) If additionally $H$ is completely reduced and $G$ is as above then $\bar{U}_{u_{z}, u^{\prime}}=0$ for all $z \in[\rho]$ and $u^{\prime}<u_{z}$, where $\bar{U}^{(H * G)}=\left[\bar{U}_{u, u^{\prime}}\right]$; hence, the block lower unitriangular matrix $U:=\bar{U}^{-1}$, for $\bar{U}:=\bar{U}^{(H * G)}$, is equal to the block matrix $U^{(H * G)}=\left[U_{u, u^{\prime}}\right]_{u, u^{\prime} \in[a]}$, with the blocks $U_{u, u^{\prime}} \in \mathbb{M}_{\sigma_{u} \times \sigma_{u^{\prime}}}(R)$ given by the formulas $U_{u, u^{\prime}}=-\bar{U}_{u, u^{\prime}}$, for $u>u^{\prime}$.

Proof Follows by applying block row Gaussian elimination arguments.

Corollary Assume that $H$ is completely reduced.

(i) If $G(z)=I_{\tau_{z}}$, for all $z \in[\rho]$, (e.g. if $G_{u_{z}, z}=X_{\tau_{z}}$; resp., $G_{u_{z}, z}=Y_{\tau_{z}}, G_{u_{z}, z}=I_{\tau_{z}}$ ) then $D=U \cdot(H * G)$ is a quasi-diagonal form of $H * G$ and the only nontrivial blocks in the matrices $U=\left[U_{u, u^{\prime}}\right]_{u, u^{\prime} \in[a]}$ and $\bar{U}=\left[\bar{U}_{u, u^{\prime}}\right]_{u, u^{\prime} \in[a]}$ as in (c), are given by the formulas $U_{u, u^{\prime}}=\left[-h_{u, z} \cdot G_{u, z} \mid 0\right]=-\bar{U}_{u, u^{\prime}}$ (resp. $U_{u, u^{\prime}}=[0 \mid-$ $\left.\left.h_{u, z} \cdot G_{u, z}\right]=-\bar{U}_{u, u^{\prime}}, U_{u, u^{\prime}}=-h_{u, z} \cdot G_{u, z}=-\bar{U}_{u, u^{\prime}}\right)$.

(ii) Let $G, U, \bar{U}$ be as in (c) and $G^{\prime}=\left[G_{u, w}^{\prime}\right]_{u \in[a], w \in[b]}$ in $\mathbb{M}_{\sigma^{\prime} \times \tau^{\prime}}(k)$ be another block matrix as in (b), with the blocks $G_{u, w}^{\prime} \in \mathbb{M}_{\sigma_{u}^{\prime} \times \tau_{w}^{\prime}}(k)$, where $\sigma^{\prime}:=\sum_{u=1}^{a} \sigma_{u}^{\prime}$ and $\tau^{\prime}:=\sum_{w=1}^{b} \tau_{w}^{\prime}$; moreover, $\bar{U}^{\prime}:=\bar{U}^{\left(H * G^{\prime}\right)}$. Then for any block diagonal matrix $E=\left[E_{u, u^{\prime}}\right]_{u, u^{\prime} \in[a]}$ in $\mathbb{M}_{\sigma \times \sigma^{\prime}}(R)$ with the blocks $E_{u, u^{\prime}} \in \mathbb{M}_{\sigma_{u} \times \sigma_{u^{\prime}}^{\prime}}(R)$, the block matrix $W:=U E \bar{U}^{\prime}$ is given by the blocks $W_{u, u^{\prime}} \in \mathbb{M}_{\sigma_{u} \times \sigma_{u^{\prime}}}(R)$, which are described as follows: $W_{u, u}=E_{u, u}$, for $u \in[a] ; W_{u, u_{z}}=U_{u, u_{z}} E_{u_{z}, u_{z}}+E_{u, u} \bar{U}_{u, u_{z}}^{\prime}$, for $u \neq u_{1}, \ldots, u_{r}$ and $z \in[\rho]$; and $W_{u, u^{\prime}}=0$, otherwise.

Proof The first assertion is straightforward, the second one follows only by the general shape of the multiplied matrices.

Remark Assume that $G=\left[G_{u, u^{\prime}}\right]$ and $G^{\prime}=\left[G_{u, u^{\prime}}^{\prime}\right]$ are as in (i), $U=U^{(H * G)}=$ $\left[U_{u, u^{\prime}}\right]$ and $\bar{U}^{\prime}=\bar{U}^{\left(H * G^{\prime}\right)}=\left[\bar{U}_{u, u^{\prime}}^{\prime}\right], E=\left[E_{u, u^{\prime}}\right]$ as in (ii) and $W:=U E \bar{U}^{\prime}=\left[W_{u, u^{\prime}}\right]$. Then the block matrix $\breve{W}=\left[\breve{W}_{u, u^{\prime}}\right]$ defined as the submatrix of the matrix $W$ with coordinates in the set $\left([\sigma] \backslash\left\{\iota_{1}, \ldots, \iota_{g}\right\}\right) \times\left(\left[\sigma^{\prime}\right] \backslash\left\{\iota_{1}^{\prime}, \ldots, \iota_{g^{\prime}}^{\prime}\right\}\right)$, where $\sigma:=\sum_{u=1}^{a} \sigma_{u}$, $\sigma^{\prime}:=\sum_{u=1}^{a} \sigma_{u}^{\prime}$ and $\iota_{1}, \ldots, \iota_{g}$, respectively $\iota_{1}^{\prime}, \ldots, \iota_{g^{\prime}}^{\prime}$ are row indices of all elements of the quasi-diagonals in $D:=D^{(H * G)}$ and $D^{\prime}:=D^{(H * G)}$, are given as follows: $\breve{W}_{u, u^{\prime}}=W_{u, u^{\prime}}$ for $u, u^{\prime} \neq u_{1}, \ldots, u_{\rho} ; \breve{W}_{u_{z}, u^{\prime}}$ is an empty matrix if $G_{u_{z}, z}=I_{\tau_{z}}$, and is the first (resp. the last) row of $W_{u_{z}, u^{\prime}}$ if $G_{u_{z}, z}=Y_{\tau_{z}}$ (resp. $G_{u_{z}, z}=X_{\tau_{z}}$ ), for $z \in[\rho]$ and $u^{\prime} \neq u_{1}, \ldots, u_{\rho}$; dually, $\breve{W}_{u, u_{z}}$ is an empty matrix if $G_{u_{z}, z}^{\prime}=I_{\tau_{z}^{\prime}}$, and is the first (resp. the last) column of $W_{u, u_{z}}$ if $G_{u_{z}, z}^{\prime}=Y_{\tau_{z}^{\prime}}\left(\operatorname{resp} . G_{u_{z}, z}^{\prime}=X_{\tau_{z}^{\prime}}\right)$, for $z \in[\rho]$ and $u \neq u_{1}, \ldots, u_{\rho}$; finally, $\breve{W}_{u_{z}, u_{z^{\prime}}}$ is an empty matrix if $G_{u_{z}, z}=I_{\tau_{z}}$ or $G_{u_{z^{\prime}}, z^{\prime}}^{\prime}=I_{\tau_{z^{\prime}}^{\prime}}$, and is 
$1 \times 1$-matrix consisting of the respective corner element of $W_{u_{z}, u_{z^{\prime}}}$ if $G_{u_{z}, z}=X_{\tau_{z}}$ (or $\left.Y_{\tau_{z}}\right)$ and $G_{u_{z^{\prime}}, z^{\prime}}^{\prime}=X_{\tau_{z^{\prime}}}\left(\right.$ or $\left.Y_{\tau_{z^{\prime}}^{\prime}}\right)$, for $z, z^{\prime} \in[\rho]$.

4.6

Proof of Theorem 4.1 Fix a tubular type $\mathbf{p}$ and an integer $n \in \mathbb{N}_{\geq p}$. Set $\bar{n}:=n-p$, $m:=\operatorname{quo}_{p}(\bar{n})$ and $r:=\operatorname{rem}_{p}(\bar{n})$. We start the realization of $(\mathrm{P} 1)-(\mathrm{P} 4)$ announced in the end of Section 4.3 by fixing the ordered $k$-base $\mathcal{B}_{\vec{y}}$ of $S_{\vec{y}}$, for every $\vec{y} \in \mathbf{L}_{+}$(see Lemma 4.4(a)). We will also treat in a natural way $\mathcal{B}_{\vec{y}}$ as a fixed $k[\xi]$-base of a free $k[\xi]$-module $k[\xi] \otimes_{k} S_{\vec{y}}$, for every $\vec{y} \in \mathbf{L}_{+}$. Then for any vertex $v=\vec{x}$, where $0 \leq \vec{x} \leq$ $\vec{c}$, the sets $\mathcal{B}_{v}^{0}=\mathcal{B}_{v}^{0}(n):=\bigsqcup_{i \in[t]} \bigsqcup_{j \in\left[p_{i}-1\right]} \bigsqcup_{\frac{p}{p_{i}}} \mathcal{B}_{\vec{n} \vec{x}_{t}+j \vec{x}_{i}-\vec{x}}$ and $\mathcal{B}_{v}^{1}=\mathcal{B}_{v}^{1}(n):=\bigsqcup_{p} \overline{\mathcal{B}}_{\bar{n} \vec{x}_{t}-\vec{x}}$ form canonical $k$-bases of the spaces $\hat{P}_{v}^{0}$ and $\hat{P}_{v}^{1}$, respectively, and of the free $k[\xi]$-modules $k[\xi] \otimes_{k} \hat{P}_{v}^{0}$ and $k[\xi] \otimes_{k} \hat{P}_{v}^{1}$, since $\hat{P}_{v}^{0}=\bigoplus_{i \in[t]} \bigoplus_{j \in\left[p_{i}-1\right]} \bigoplus_{\frac{p}{p_{i}}} S_{\vec{n}_{x_{t}}+j \vec{x}_{i}-\vec{x}}$ and $\hat{P}_{v}^{1}=\bigoplus_{p} S_{\vec{n} \vec{x}_{t}-\vec{x}}$. Note that both, $\mathcal{B}_{v}^{0}$ and $\mathcal{B}_{v}^{1}$, are naturally equipped with the ordering induced by $\kappa_{\mathbf{p}}$, so (P1) is finished.

Next, according to (P2), we construct, for any $v$ as above, the matrix $F_{v}=F_{v}^{(n)}$ of the $k[\xi]$-homomorphism $N_{v}^{(n)}$ with respect to the bases $\mathcal{B}_{v}^{0}$ and $\mathcal{B}_{v}^{1}$. Observe that the matrix $G_{v}$ of the $k$-linear map $\hat{P}_{v}^{1} \rightarrow \hat{P}_{v}^{0}$ with the coordinates defined by the appropriate multiplications $X_{i}^{j} \cdot: S_{\vec{n}_{t}-\vec{x}} \rightarrow S_{\vec{n} \vec{x}_{t}+j \vec{x}_{i}-\vec{x}}$, (with respect to $\mathcal{B}_{v}^{0}$, and $\mathcal{B}_{v}^{1}$ ) is given by the blocks $G_{v ; u, w}:=\Xi_{\vec{y}(\bar{n}, \vec{x}) ; i, j}$, for $w \in[b]$ and $u \in[a]$, where $a$ is the length of $\kappa_{\mathbf{p}}, j \vec{x}_{i}$ is a $u$ th element of $\kappa_{\mathbf{p}}$ and $b:=p$ (see Section 4.4). Furthermore, $F_{v}=N * G_{v}$, where $N=N_{\mathbf{p}}$. In particular this implies that "the configuration of the types of matrix" appearing as the respective blocks $F_{v ; u, w}=F_{v ; u, w}^{(n)}$ of $F_{v}$, for $u, w$, "depends on $n$ periodically modulo $p$ " and we can easily determine them precisely in each of the cases.

To find for any $v$ the invertible $\xi$-matrix $U_{v}=U_{v}^{(n)}$ and the quasi-diagonal form $D_{v}=D_{v}^{(n)}$ of $F_{v}$, note that all $\xi$-matrices $N_{\mathbf{p}}$ are completely reduced with $\rho\left(=\rho_{\mathbf{p}}\right)$ equal to $b$ and that the sequence $\underline{u}=\underline{u}_{\mathbf{p}}=\left(u_{1}, \ldots, u_{\rho}\right)$ is equal to $(1,2)$, respectively, $(1,2,4),(1,2,4,5)$ and $(1,2,3,5,6,7)$, for the consecutive tubular types $\mathbf{p}=(2,2,2,2),(3,3,3),(2,4,4)$ and $(2,3,6)$. Moreover, for any $v$, among the blocks $G_{v ; u_{z}, z}=G_{v ; u_{z}, z}^{(n)}$ of $G_{v}$, for $z \in[\rho]$, it can appear only $I_{l}, X_{l}$ and $Y_{l}$ ("with the periodic dependence on $n$ modulo $p$ "), since for any $\mathbf{p}$, the vertices from the sequence $\kappa_{\mathbf{p}}$, which correspond to $u_{1}, \ldots, u_{\rho}$, lie on the two upper arms of the quiver $Q$ (see the formulas from Lemma 4.4(b)). Consequently, for any $v$ all the assumptions of Lemma 4.5(b,c) and Corollary 4.5.(i) are satisfied. Hence, setting $U_{v}=U_{v}^{(n)}:=$ $U^{\left(F_{v}\right)}$ and $D_{v}=D_{v}^{(n)}:=D^{\left(F_{v}\right)}$, where $F_{v}=F_{v}^{(n)}$, we automatically have the following: $D_{v}=U_{v} \cdot F_{v}, \quad U_{v}^{-1}=\bar{U}_{v}^{(n)}$, where $\bar{U}_{v}^{(n)}:=\bar{U}^{\left(F_{v}\right)}$, and $D_{v}$ is a quasi-diagonal form of $F_{v}$ with the quasi-diagonal formed by quasi-diagonals of the matrices $I_{l}, X_{l}$ and $Y_{l}$. Due to the formulas defining $D_{v}, U_{v}$ and $\bar{U}_{v}$ "the configuration of the types of matrices" appearing as the respective blocks in these matrices, in particular, the blocks $U_{v ; u, u^{\prime}}=U_{v ; u, u^{\prime}}^{(n)}$ of $U_{v}$ and $\bar{U}_{v ; u, u^{\prime}}=U_{v ; u, u^{\prime}}^{(n)}$ of $U_{v}$ also "depends on $n$ periodically modulo $p$ " and we can easily determine them in each of the cases.

Now we pass to (P3). It is easily seen that the $\xi$-matrix $E_{\alpha}=E_{\alpha}^{(n)}=\left[E_{\alpha ; u, u^{\prime}}\right]_{u, u^{\prime} \in[a]}$ for an arbitrary arrow $\alpha=\alpha_{j^{\prime}}^{\left(i^{\prime}\right)}$ is always a block diagonal matrix. Since $\hat{P}_{\alpha}^{0}$ is a direct sum of the multiplications $X_{i^{\prime}}: S_{\vec{n}_{t}+j \vec{x}_{i}-j^{\prime} \vec{x}_{i^{\prime}}} \rightarrow S_{\vec{n} \vec{x}_{t}+j \vec{x}_{i}-\left(j^{\prime}-1\right) \vec{x}_{i^{\prime}}}$ for $i \in[t]$ and $j \in\left[p_{i}-1\right]$, the blocks on the diagonal are given by the formula $E_{\alpha ; u, u}=\Xi_{\vec{y}(\bar{n}, \vec{x}) ; i^{\prime}, 1}$, 
for $\vec{x}=j^{\prime} \vec{x}_{i^{\prime}}-j \vec{x}_{i}$, where $j \vec{x}_{i}$ is the vertex which corresponds to $u \in[a]$. Consequently, "the configuration of the types of matrices" appearing as the respective blocks again "depends on $n$ periodically modulo $p$ " and we can easily determine them precisely in each of the cases.

Now, due to the previous information, we can form the block matrices $W_{\alpha}=$ $W_{\alpha}^{(n)}=\left[W_{\alpha ; u, u^{\prime}}\right]_{u, u^{\prime} \in[a]}$ such that $W_{\alpha}^{(n)}:=U_{s(\alpha)} E_{\alpha} \bar{U}_{t(\alpha)}$, where $U_{s(\alpha)}=U_{s(\alpha)}^{(n)}$ and $\bar{U}_{t(\alpha)}=\bar{U}_{t(\alpha)}^{(n)}$, applying the formulas from Corollary 4.5(ii). Namely for the only nontrivial block coordinates for $W_{\alpha}$ we have $W_{u, u_{z}}=U_{u, u_{z}} E_{u_{z}, u_{z}}+E_{u, u} \bar{U}_{u, u_{z}}^{\prime}$, for $u \neq$ $u_{1}, \ldots, u_{\rho}$ and $z \in[\rho]$, where $W_{u, u_{z}}:=W_{\alpha ; u, u_{z}}, E_{u_{z}, u_{z}}:=E_{\alpha ; u_{z}, u_{z}}, U_{u, u_{z}}:=U_{s(\alpha) ; u, u_{z}}$ and $\bar{U}_{u, u_{z}}^{\prime}:=\bar{U}_{t(\alpha) ; u, u_{z}}$. A simple analysis of the results obtained by an application of the formulas from Lemma 4.4 and Corollary 4.5(i) in the previous steps shows that the blocks $U_{u, u_{z}}, \bar{U}_{u, u_{z}}^{\prime}$ have one of the following "shapes": $\pm I, \pm \xi \cdot I, \pm Y, \pm \xi \cdot Y$, $\pm Z, \pm \xi \cdot Z, \pm Z^{\lambda}, \pm X^{\mathrm{tr}}, \pm \xi \cdot X^{\mathrm{tr}}, \pm Y^{\mathrm{tr}}, \pm[Z \mid 0], \pm[\xi \cdot Z \mid 0], \pm[0 \mid Z], \pm\left[Z^{\lambda} \mid 0\right]$, $\pm\left[0 \mid Z^{\lambda}\right]$ (here 0 denotes the zero column), whereas $E_{u, u}$ (in particular $E_{u_{z}, u_{z}}$ ) the shapes $I, X, Y, Z$ or $Z^{\lambda}$. Moreover, it is not hard to check that the nonzero $W_{u, u_{z}}$ 's can only appear in the following eight cases:

\begin{tabular}{l|cccccccc}
$U_{u, u_{z}}$ & $I_{m+1}$ & $\xi \cdot I_{m+1}$ & $I_{m+1}$ & $Z_{m+1}$ & $\xi \cdot Z_{m+1}$ & $Z_{m+1}$ & $Z_{m+1}^{\lambda}$ & $Z_{m+1}^{\lambda}$ \\
$E_{u_{z}, u_{z}}$ & $I_{m+1}$ & $I_{m+1}$ & $I_{m+1}$ & $I_{m+1}$ & $I_{m+1}$ & $I_{m+1}$ & $I_{m+1}$ & $I_{m+1}$ \\
$E_{u, u}$ & $X_{m}$ & $X_{m}$ & $Y_{m}$ & $X_{m+1}$ & $X_{m+1}$ & $Y_{m+1}$ & $X_{m+1}$ & $Y_{m+1}$ \\
$\bar{U}_{u, u_{z}}^{\prime}$ & $-X_{m}^{\mathrm{tr}}$ & $-\xi \cdot X_{m}^{\mathrm{tr}}$ & $-Y_{m}^{\mathrm{tr}}$ & $-\left[Z_{m} \mid 0\right]$ & $-\left[\xi \cdot Z_{m} \mid 0\right]$ & $-\left[0 \mid Z_{m}\right]$ & $-\left[Z_{m}^{\lambda} \mid 0\right]$ & $-\left[0 \mid Z_{m}^{\lambda}\right.$ \\
\hline$W_{u, u_{z}}$ & $0_{m} \oplus[1]$ & $0_{m} \oplus[\xi][1] \oplus 0_{m}$ & $0_{m} \oplus\left[\begin{array}{l}1 \\
1\end{array}\right]$ & $0_{m} \oplus\left[\begin{array}{l}\xi \\
\xi\end{array}\right]\left[\begin{array}{l}1 \\
1\end{array}\right] \oplus 0_{m}$ & $0_{m} \oplus\left[\begin{array}{l}1 \\
\lambda\end{array}\right]\left[\begin{array}{l}1 \\
\lambda\end{array}\right] \oplus 0_{m}$
\end{tabular}

where $0_{m}$ denotes the zero $m \times m$-matrix and $A \oplus A^{\prime}$ the matrix $\left[\begin{array}{cc}A & 0 \\ 0 & A^{\prime}\end{array}\right]$, for $\xi$ matrices $A$ and $A^{\prime}$. Consequently, "the configuration of the types of matrices" appearing as the blocks of matrices $W_{\alpha}$ again "depends on $n$ periodically modulo $p$ " and we can easily determine them precisely in each of the cases.

To form in step (P4), for any arrow $\alpha=\alpha_{j}^{(i)}$, the submatrix $\breve{W}_{\alpha}=\breve{W}_{\alpha}^{(n)}$ of $W_{\alpha}$, we apply Remark 4.5 in each case determined by $r$. Observe that since the positions of the indices determining the quasi-diagonals inside the diagonal blocks in $D_{s(\alpha)}^{(n)}$ and $D_{t(\alpha)}^{(n)}$ depend in fact on $r$, so by the property of the matrices $W_{\alpha}^{(n)}$ formulated above, the same holds true for "the configuration of the types of matrices" appearing as the blocks for the resulting matrices $W_{\alpha}^{(n)}$.

To complete the proof, notice that performing all the described processes in each of the cases we obtain exactly all matrix bimodules from Section 4.7.

For better illustration of the presented construction we provide below an example, showing how to obtain for one data collection $(\mathbf{p}, r)$ and a fixed arrow $\alpha$, the matrix $\breve{W}_{\alpha}^{(n)}\left(=B_{\alpha}^{(n)}\right)$, for any $m \in \mathbb{N}$, where $\bar{n}=n-p=m p+r$.

Example Let $\mathbf{p}:=(3,3,3), r:=1$ and $\alpha:=\alpha_{1}^{(1)}$ (the situation for this arrow is the most complicated one for $(3,3,3)$ and moreover, fully representative for all other p's). Then $t=3, p=3, s(\alpha)=0, t(\alpha)=\vec{x}_{1}$.

Fix an arbitrary $n \in \mathbb{N}_{\geq 3}$ and set $\bar{n}=n-3, m=\mathrm{quo}_{3}(\bar{n})$ (so then $\bar{n}=3 m+1$ ). Below we use the notation as in the proof above and also that from Section 4.7. By 0 we denote the zero scalar or the zero block matrix of respective size depending on the context. 


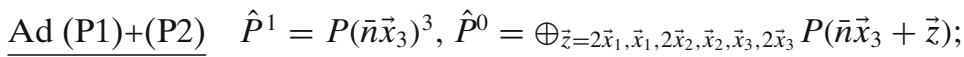

$\left(k[\xi] \otimes_{k} \hat{P}^{1}\right)_{0} \cong k[\xi]^{3(m+1)}, \quad\left(k[\xi] \otimes_{k} \hat{P}^{0}\right)_{0} \cong k[\xi]^{5(m+1)+(m+2)}$,

$\left(k[\xi] \otimes_{k} \hat{P}^{1}\right)_{\vec{x}_{1}} \cong k[\xi]^{3 m}, \quad\left(k[\xi] \otimes_{k} \hat{P}^{0}\right)_{\vec{x}_{1}} \cong k[\xi]^{2(m+1)+3 m+(m+1)}$;

$F_{0}=\left[\begin{array}{ccc}\dot{I} & 0 & 0 \\ 0 & \dot{I} & 0 \\ -\dot{I} & -\xi \dot{I} & 0 \\ 0 & 0 & \dot{I} \\ -\dot{I} & -\dot{I} & -\dot{I} \\ -\dot{Z} & 0 & 0\end{array}\right], D_{0}=\left[\begin{array}{ccc}\dot{I} & 0 & 0 \\ 0 & \dot{I} & 0 \\ 0 & 0 & 0 \\ 0 & 0 & \dot{I} \\ 0 & 0 & 0 \\ 0 & 0 & 0\end{array}\right], F_{\vec{x}_{1}}=\left[\begin{array}{ccc}X & 0 & 0 \\ 0 & X & 0 \\ -I & -\xi \cdot I & 0 \\ 0 & 0 & I \\ -I & -I & -I \\ -Z & 0 & 0\end{array}\right], D_{\vec{x}_{1}}=\left[\begin{array}{ccc}X & 0 & 0 \\ 0 & X & 0 \\ 0 & 0 & 0 \\ 0 & 0 & I \\ 0 & 0 & 0 \\ 0 & 0 & 0\end{array}\right]$,

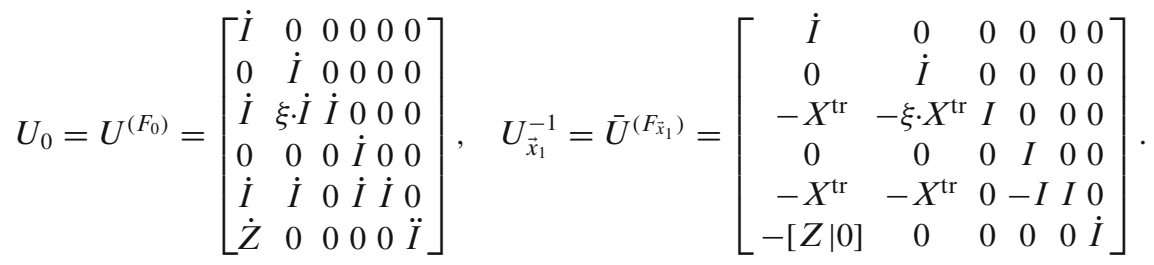

$\underline{\operatorname{Ad}(\mathrm{P} 3)+(\mathrm{P} 4)} \quad E_{\alpha}=\dot{I} \oplus \dot{I} \oplus X \oplus X \oplus X \oplus \dot{X}$,

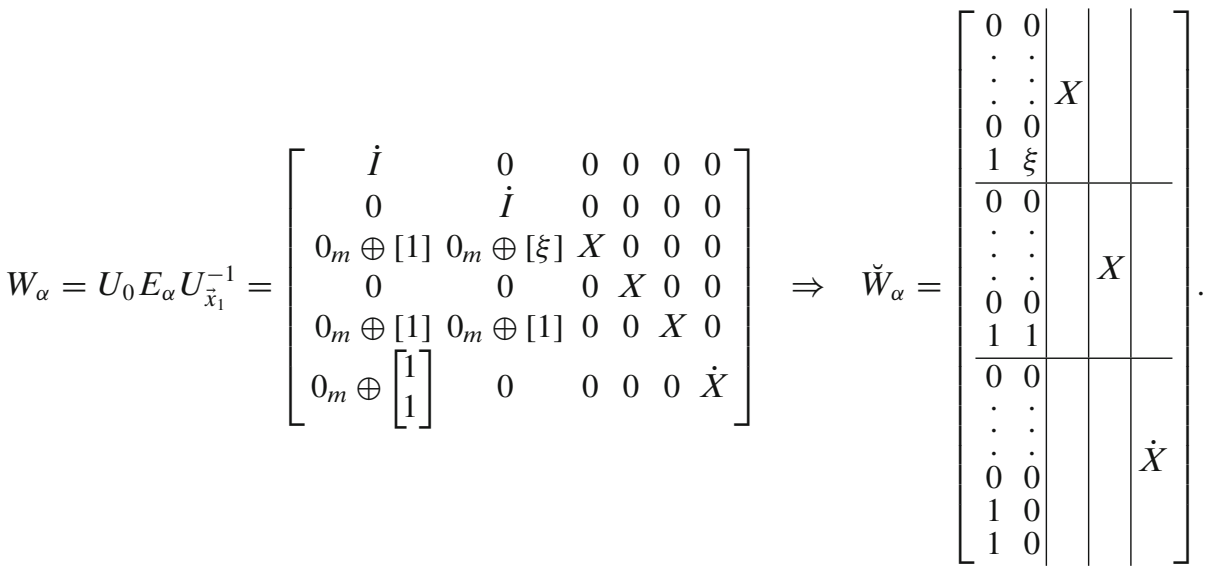

4.7

We will further use the following abbreviate notation. For a fixed $m \in \mathbb{N}$ we always write $X:=X_{m}, Y:=Y_{m}, Z:=X+Y, Z^{\lambda}=X+\lambda Y \in \mathbb{M}_{(m+1) \times m}(k)$ and $I=I_{m}$, respectively, $\dot{X}:=X_{m+1}, \dot{Y}:=Y_{m+1}, \dot{Z}:=Z_{m+1}, \dot{Z}^{\lambda}=Z_{m+1}^{\lambda} \in \mathbb{M}_{(m+2) \times(m+1)}(k)$, $\dot{I}:=I_{m+1}$ and $\ddot{I}:=I_{m+2}$.

For each tubular type $\mathbf{p}$, we give below $p$ series of the matrix bimodules $B^{(n)}$ in $\bmod _{k[\xi]}^{\text {mat }} \Lambda$, for $n \in \mathbb{N}_{\geq p}$, determined by the reminder $r:=\operatorname{rem}_{p}(n)$. We also provide for the convenience in each case the rank vectors of $B^{(n)}$ 's. For a fixed $r \in \mathbb{Z}_{p}$, we exhibit the bimodules $B^{(n)}$, for $\bar{n}(:=n-p)=m p+r$, for all $m \in \mathbb{N}$, and their rank 
vectors, using the natural table of matrices convention; the consecutive rows of the table consist of the all matrices over arrows (resp. ranks over the points) appearing on the consecutive arms in $Q_{\mathbf{p}}$ (see Section 2.1). The empty spaces in the matrices below always mean the zero blocks.

\section{The Table of the Bimodules $B^{(n)}$}

- $[2,2,2,2], r=0 ; \bar{n}=2 m$ : - $[2,2,2,2], r=1 ; \bar{n}=2 m+1$ :

$$
\left[\begin{array}{c}
1+2 m \\
1+2 m \\
(m+1)+m \\
2(m+1)(m+1) 2 m
\end{array}\right]
$$
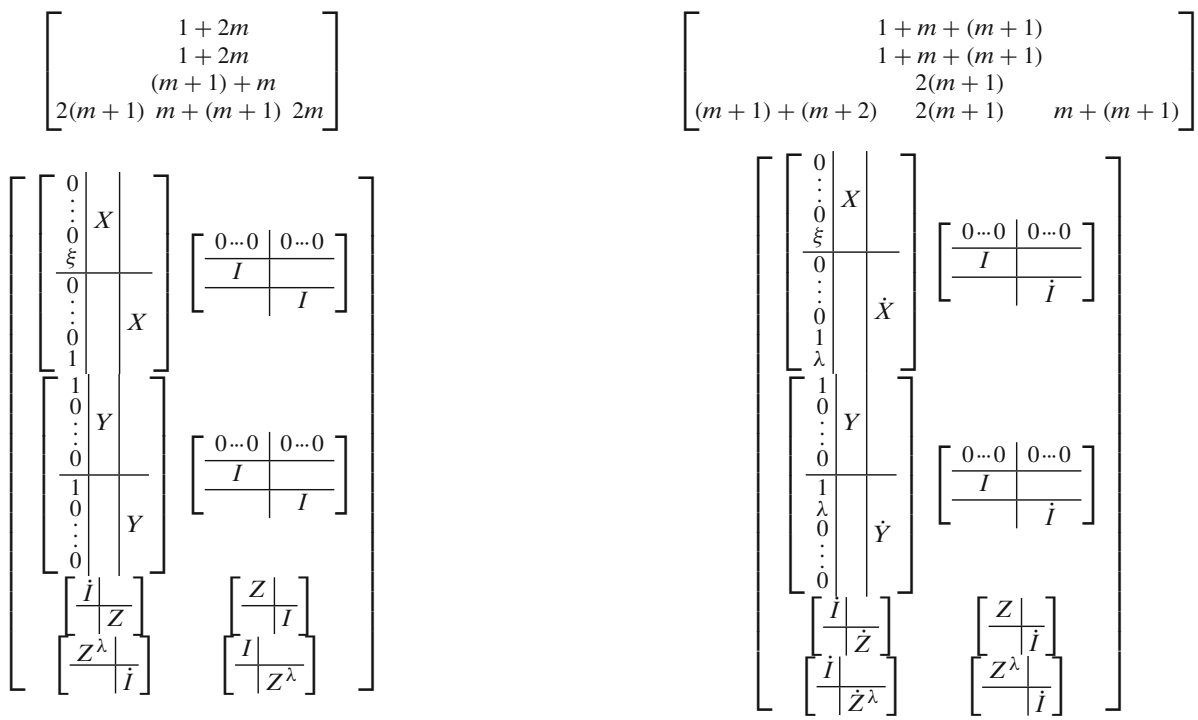

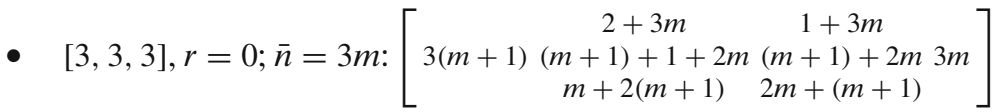

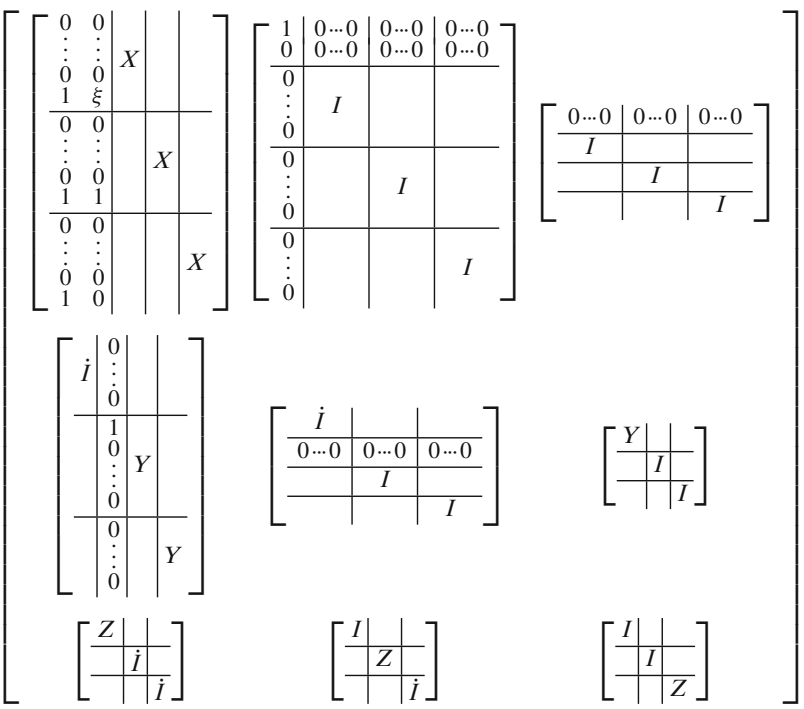


- $\quad[3,3,3], r=1$;

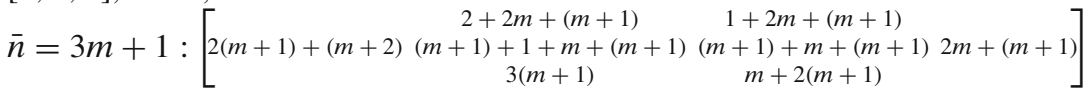

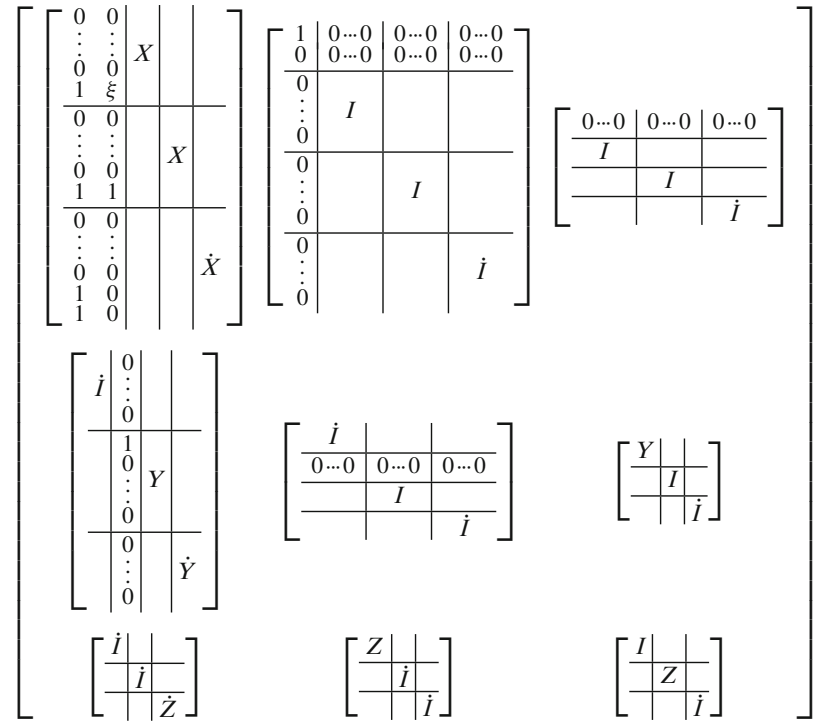

- $\quad[3,3,3], r=2$;

$\bar{n}=3 m+2:\left[\begin{array}{ccc}2+m+2(m+1) & 1+m+2(m+1) & \\ (m+1)+2(m+2) & (m+1)+1+2(m+1) & 3(m+1) \\ 2(m+1)+(m+2) & 3(m+1) & m+2(m+1)\end{array}\right]$

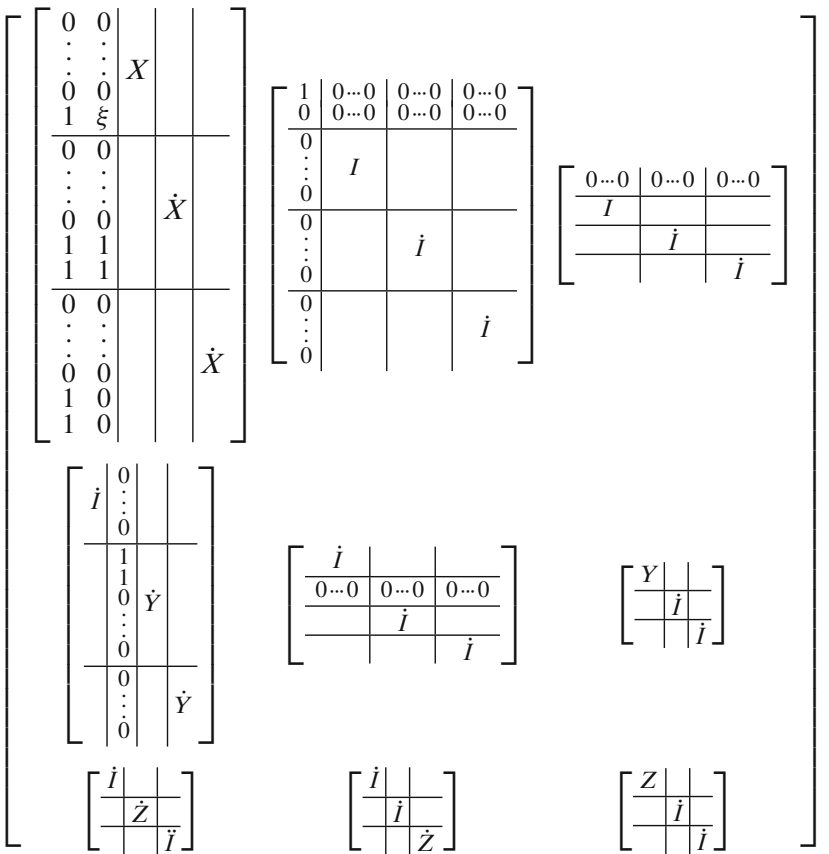


- $\quad[2,4,4], r=0$;

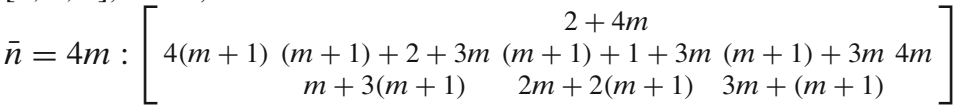

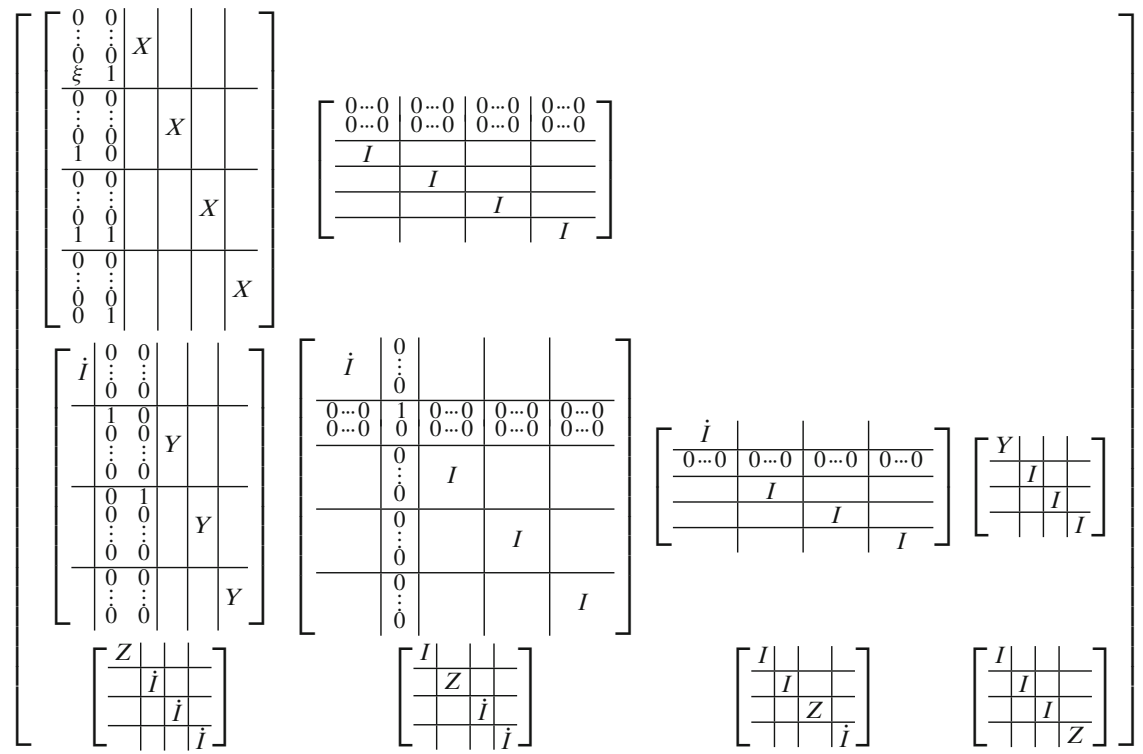

- $\quad[2,4,4], r=1 ; \bar{n}=4 m+1$ :

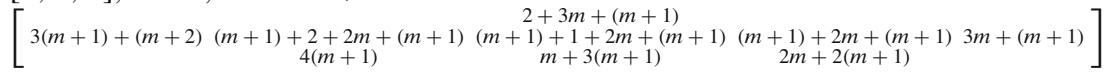
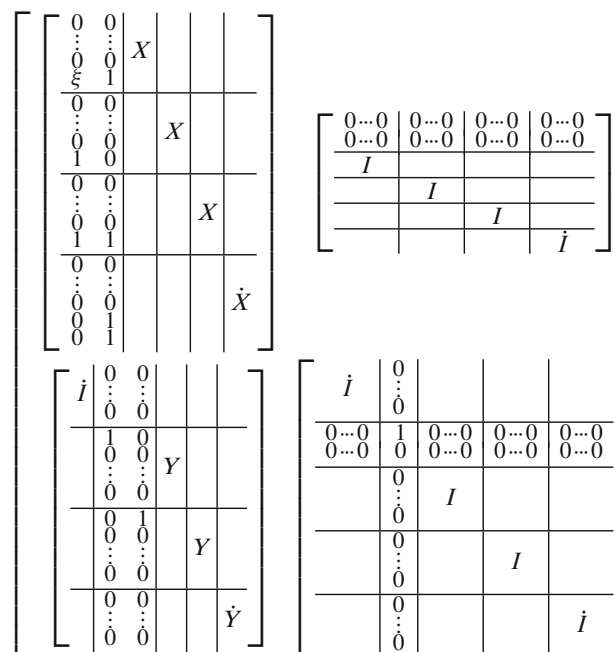

$\left[\begin{array}{c|c|c|c|c}\dot{I} & 0 & & & \\ & \dot{\vdots} & & & \\ \hline 0 \cdots 0 & 1 & \ldots \ldots 0 & 0 \cdots 0 & 0 \ldots 0 \\ 0 \cdots 0 & 0 & 0 \cdots 0 & 0 \cdots 0 & 0 \cdots 0 \\ \hline & 0 & I & & \\ & \dot{\dot{0}} & I & & \\ \hline & 0 & & & \\ & \dot{\dot{0}} & & I & \\ \hline & 0 & & & \dot{I}\end{array}\right]$
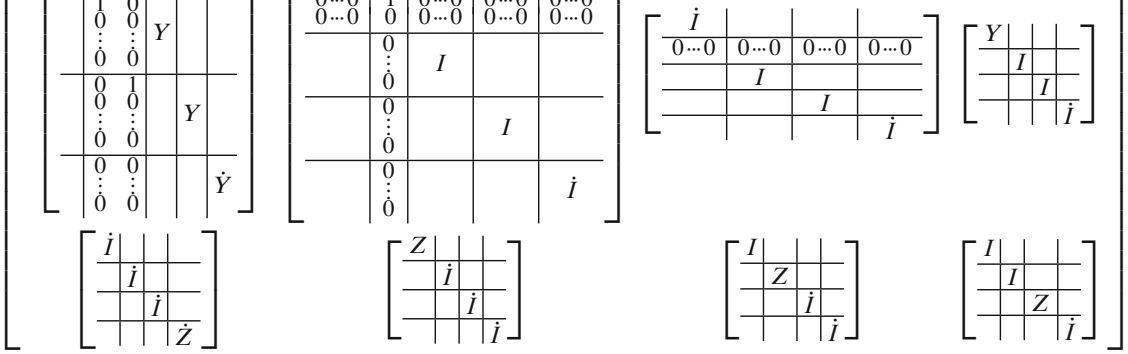
- $\quad[2,4,4], r=2 ; \bar{n}=4 m+2$ :

$\left[\begin{array}{cccc}2(m+1)+2(m+2) & (m+1)+2+m+2(m+1) & (m+1)+2(m+1) \\ 3(m+1)+(m+2) & (m+2(m+1) & (m+1)+m+2(m+1) & 2 m+2(m+1) \\ 4(m+1) & m+3(m+1)\end{array}\right]$
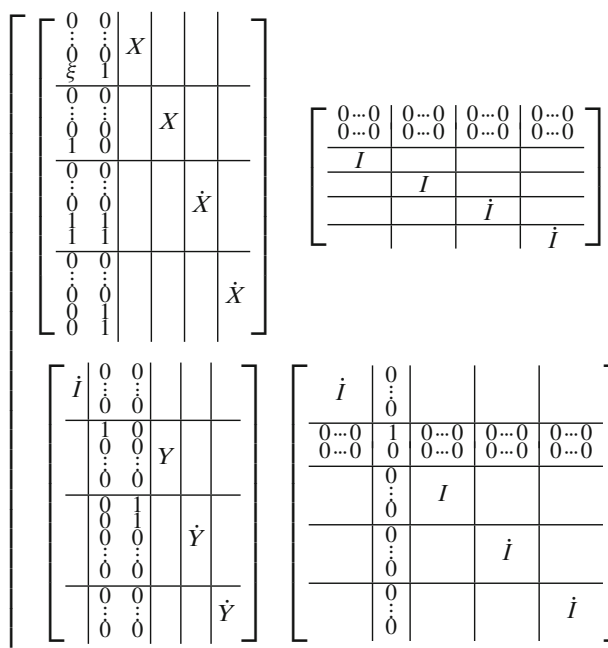

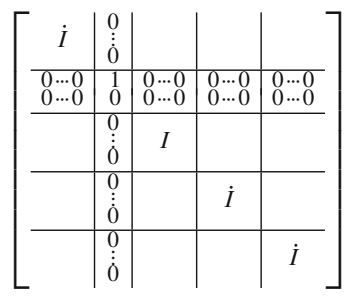
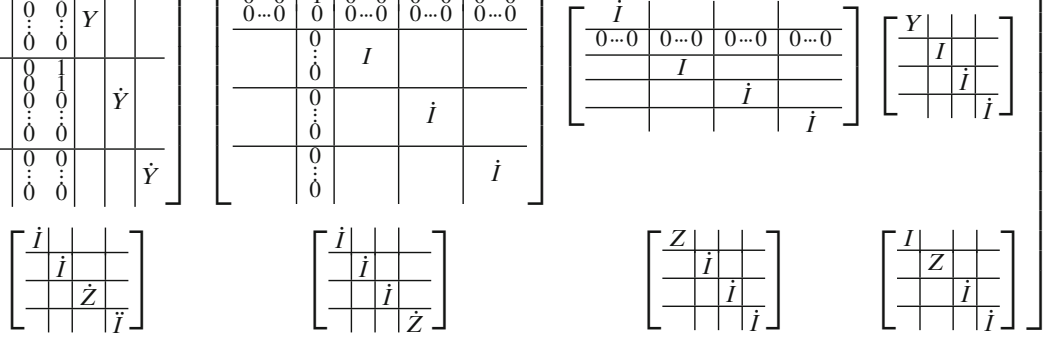

- $\quad[2,4,4], r=3 ; \bar{n}=4 m+3$ :

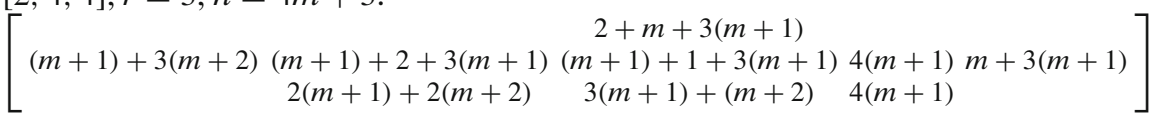
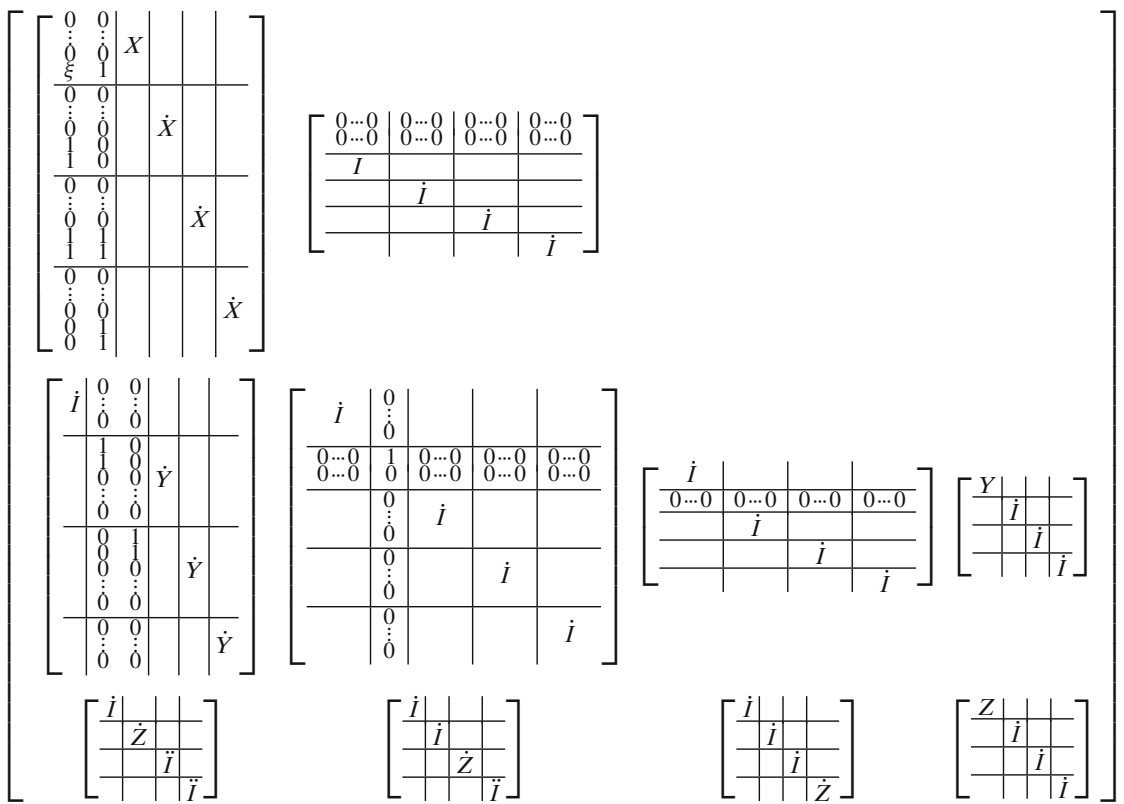
- $\quad[2,3,6], r=0 ; \bar{n}=6 m$ :

$\left[\begin{array}{llllll}6(m+1) & & (m+1)+3+5 m & 3+6 m & (m+1)+1+5 m & 6 m\end{array}\right]$

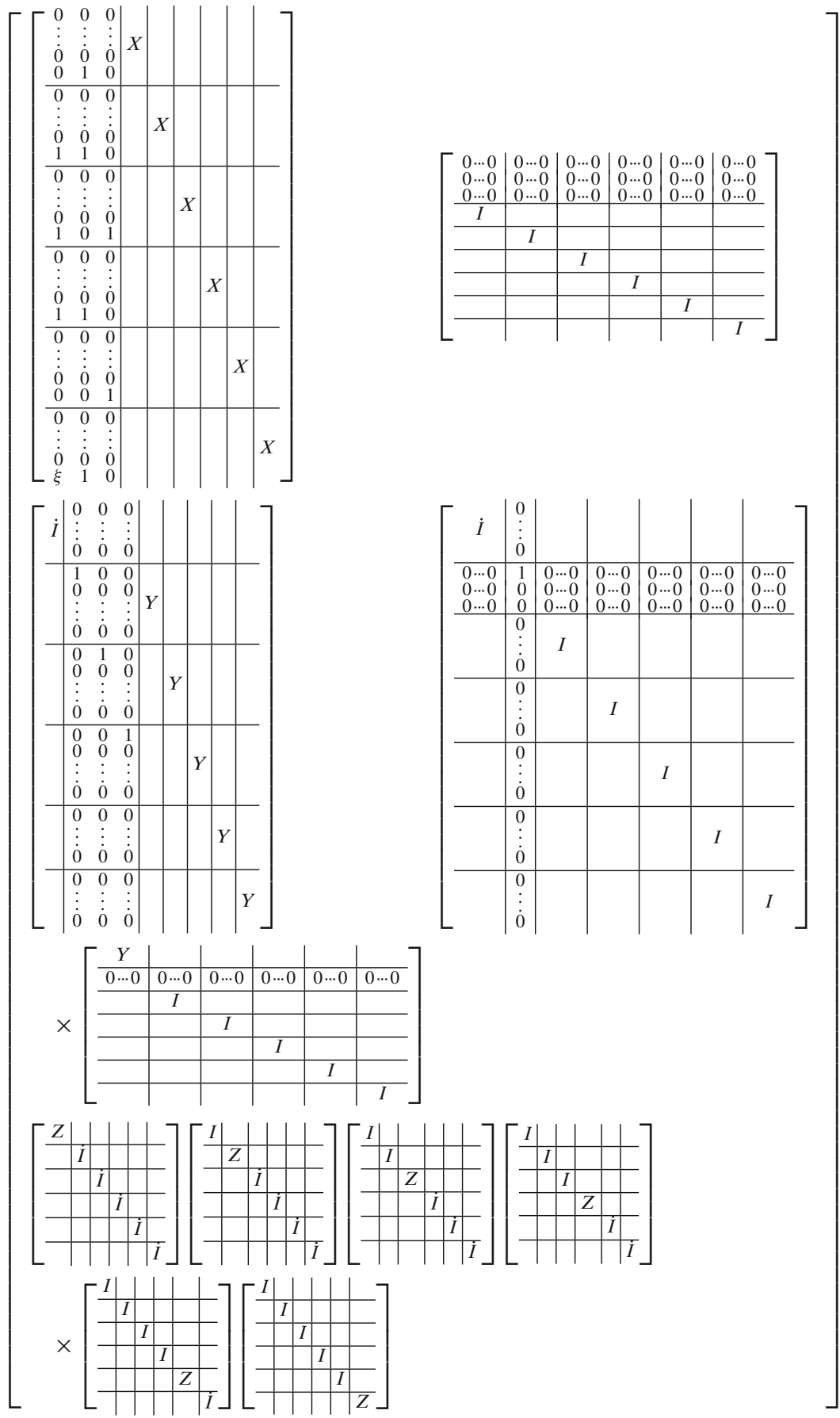


- $\quad[2,3,6], r=1 ; \bar{n}=6 m+1$ :

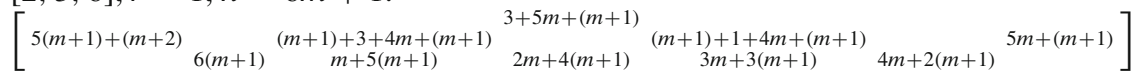
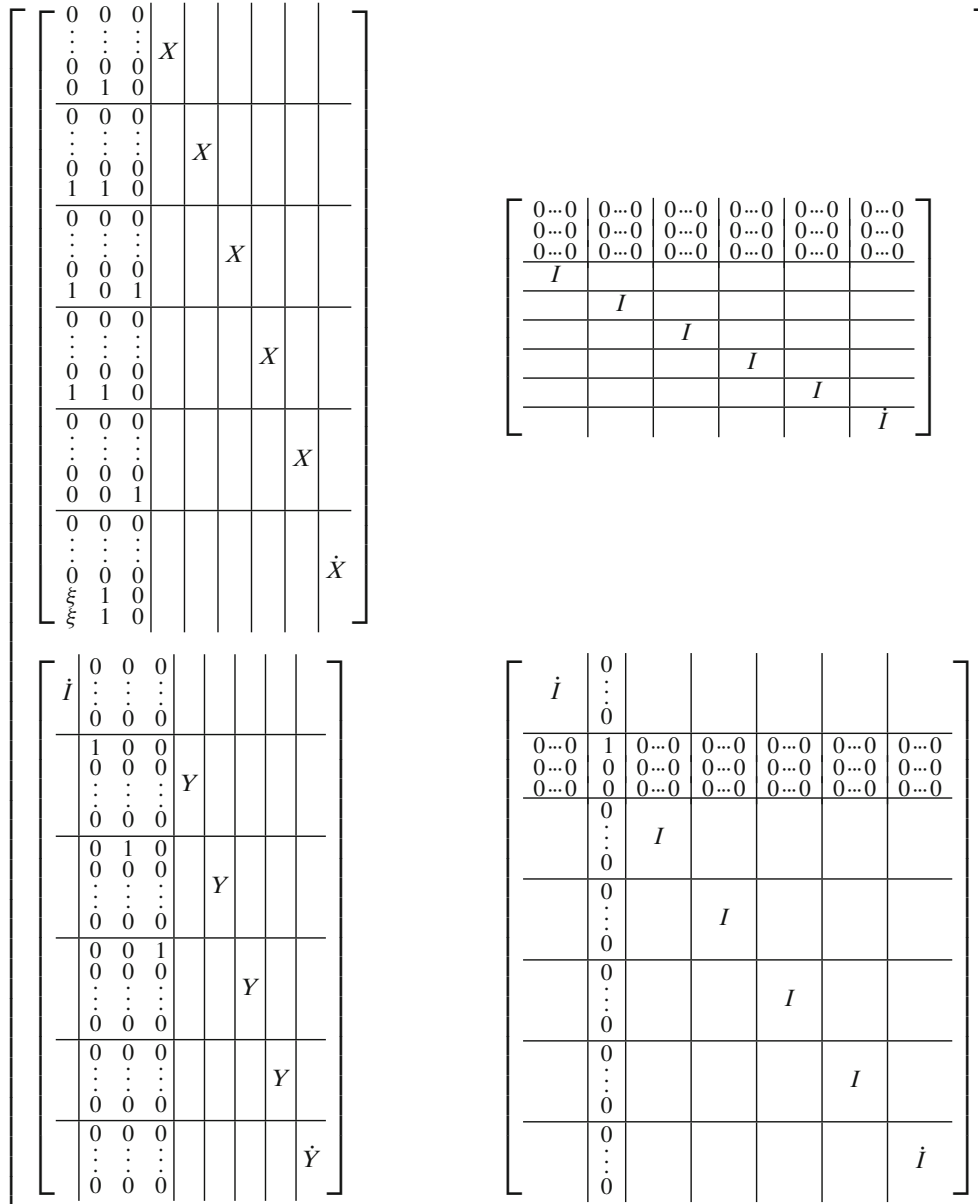

$\times\left[\begin{array}{c|c|c|c|c|c}Y & & & & & \\ \hline 0 \cdots 0 & 0 \cdots 0 & 0 \cdots 0 & 0 \cdots 0 & 0 \cdots 0 & 0 \cdots 0 \\ \hline & I & & & & \\ \hline & & I & & & \\ \hline & & & I & & \\ \hline & & & & I & \\ \hline & & & & & I\end{array}\right]$

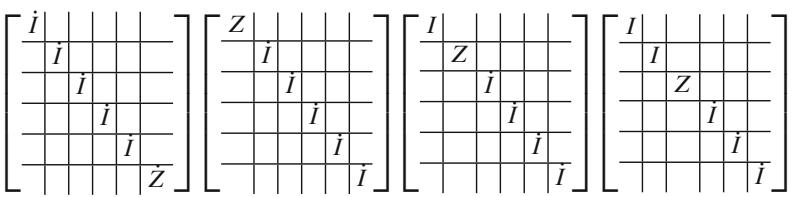

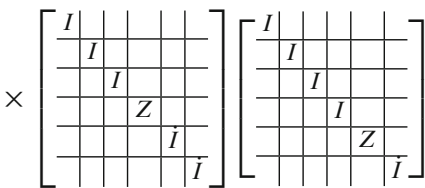


- $\quad[2,3,6], r=2 ; \bar{n}=6 m+2$ :

$\left[\begin{array}{cccc}4(m+1)+2(m+2) & & (m+1)+3+3 m+2(m+1) & 3+4 m+2(m+1) \\ 6(m+1) & m+5(m+1) & (m+1)+1+3 m+2(m+1) \\ 2 m+4(m+1) & 3 m+3(m+1)\end{array}\right]$

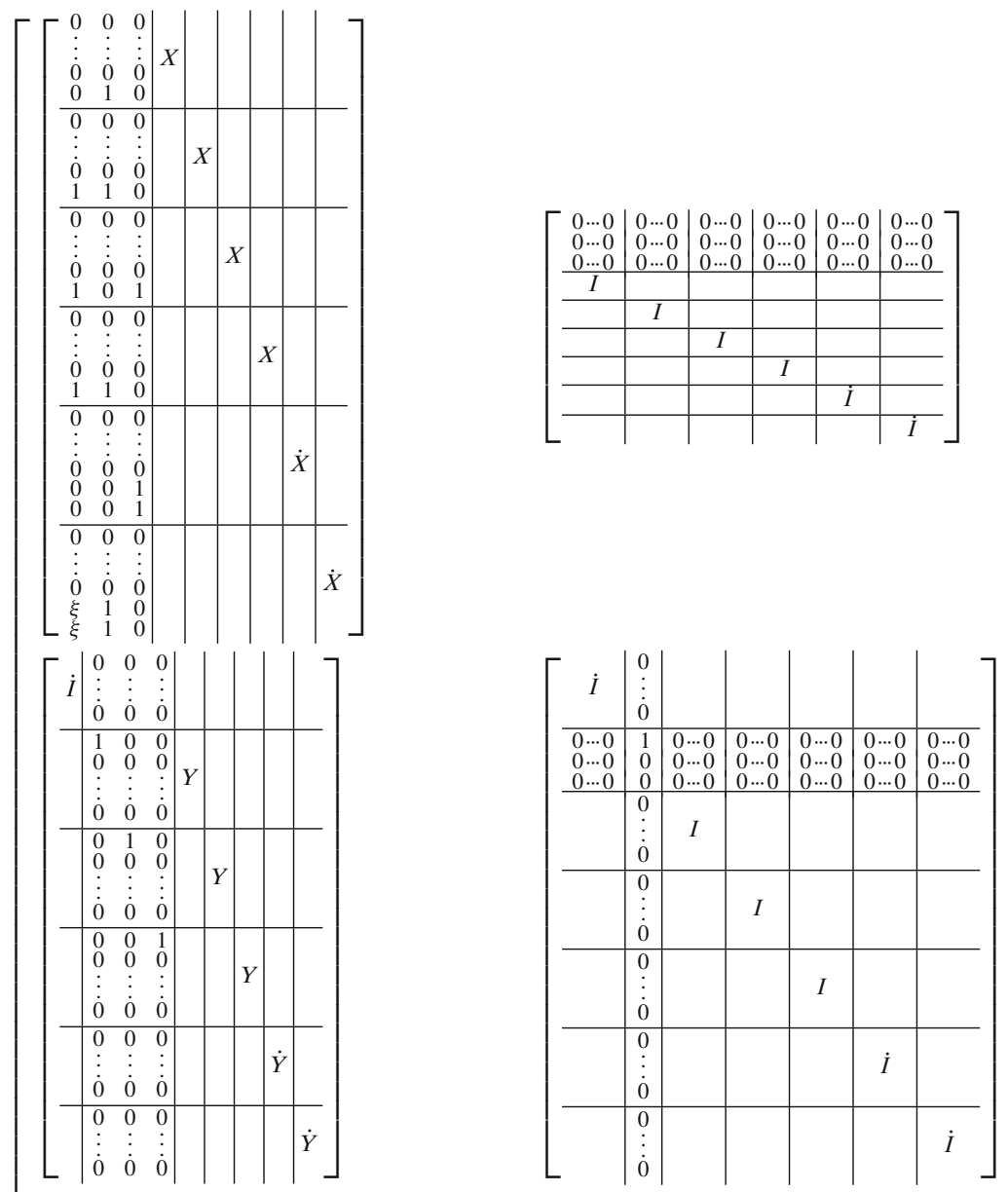

$\times\left[\begin{array}{c|c|c|c|c|c}Y & & & & & \\ \hline 0 \cdots 0 & 0 \cdots 0 & 0 \cdots 0 & 0 \cdots 0 & 0 \cdots 0 & 0 \cdots 0 \\ \hline & I & & & & \\ \hline & & I & & & \\ \hline & & & I & & \\ \hline & & & & I & I\end{array}\right]$

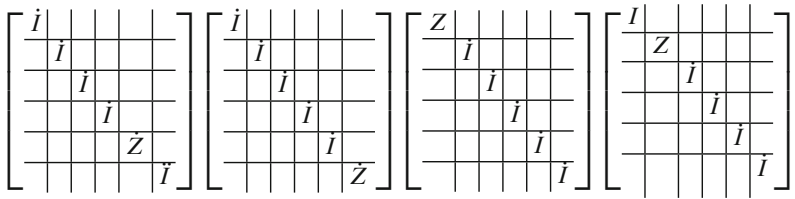

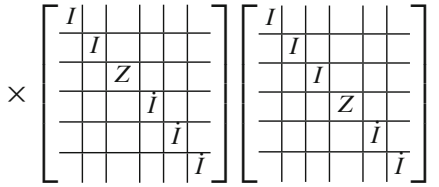


- $\quad[2,3,6], r=3 ; \bar{n}=6 m+3$ : $\left[\begin{array}{rr}3(m+1)+3(m+2) & (m+1)+3+2 m+3(m+1) \\ 4(m+1)+2(m+2) & 5(m+1)+(m+2)\end{array}\right.$

$3+3 m+3(m+1)$

$6(m+1) \quad \begin{array}{cc}(m+1)+1+2 m+3(m+1) & 3 m+3(m+1) \\ m+5(m+1) & 2 m+4(m+1)\end{array}$
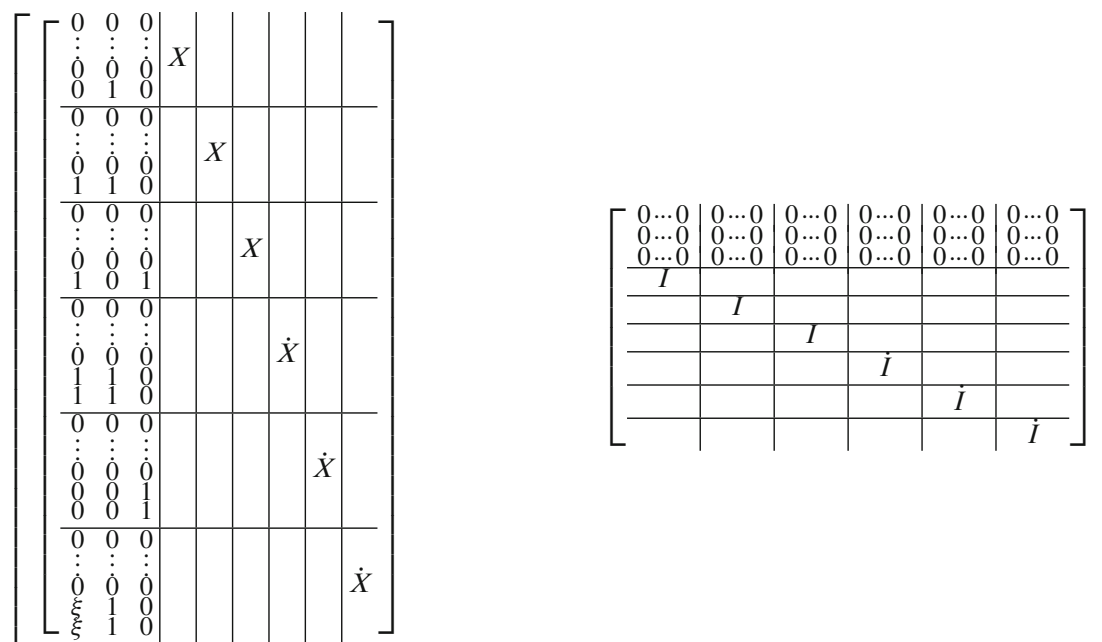

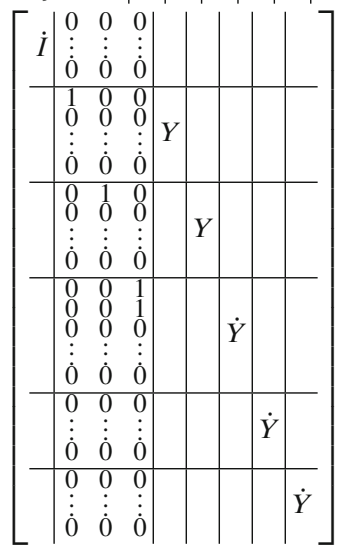

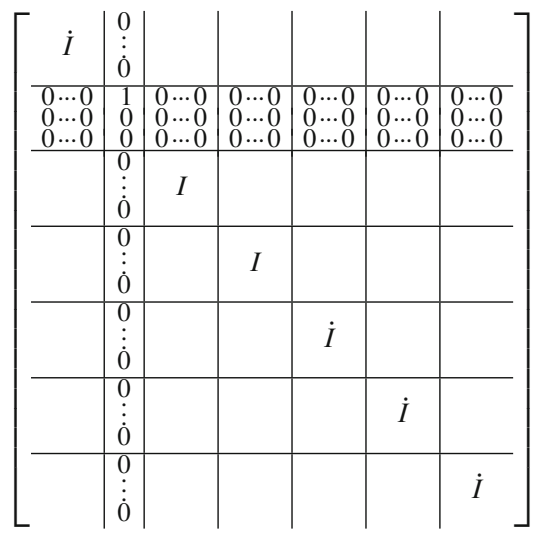

$\times\left[\begin{array}{c|c|c|c|c|c}Y & & & & & \\ \hline 0 \cdots 0 & 0 \cdots 0 & 0 \cdots 0 & 0 \cdots 0 & 0 \cdots 0 & 0 \cdots 0 \\ \hline & I & I & & & \\ \hline & & I & & & \\ \hline & & & I & & \\ \hline & & & & I & \\ \hline & & & & & I\end{array}\right]$

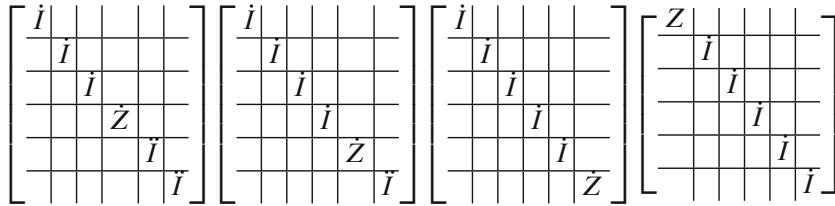

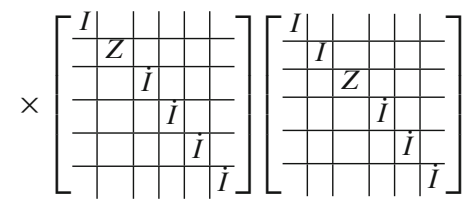


- $\quad[2,3,6], r=4 ; \bar{n}=6 m+4$ :

$\left[\begin{array}{llll}2(m+1)+4(m+2) & (m+1)+3+m+4(m+1) & 3+2 m+4(m+1) & (m+1)+1+m+4(m+1) \\ 6(m+1) & m+5(m+1)\end{array}\right]$
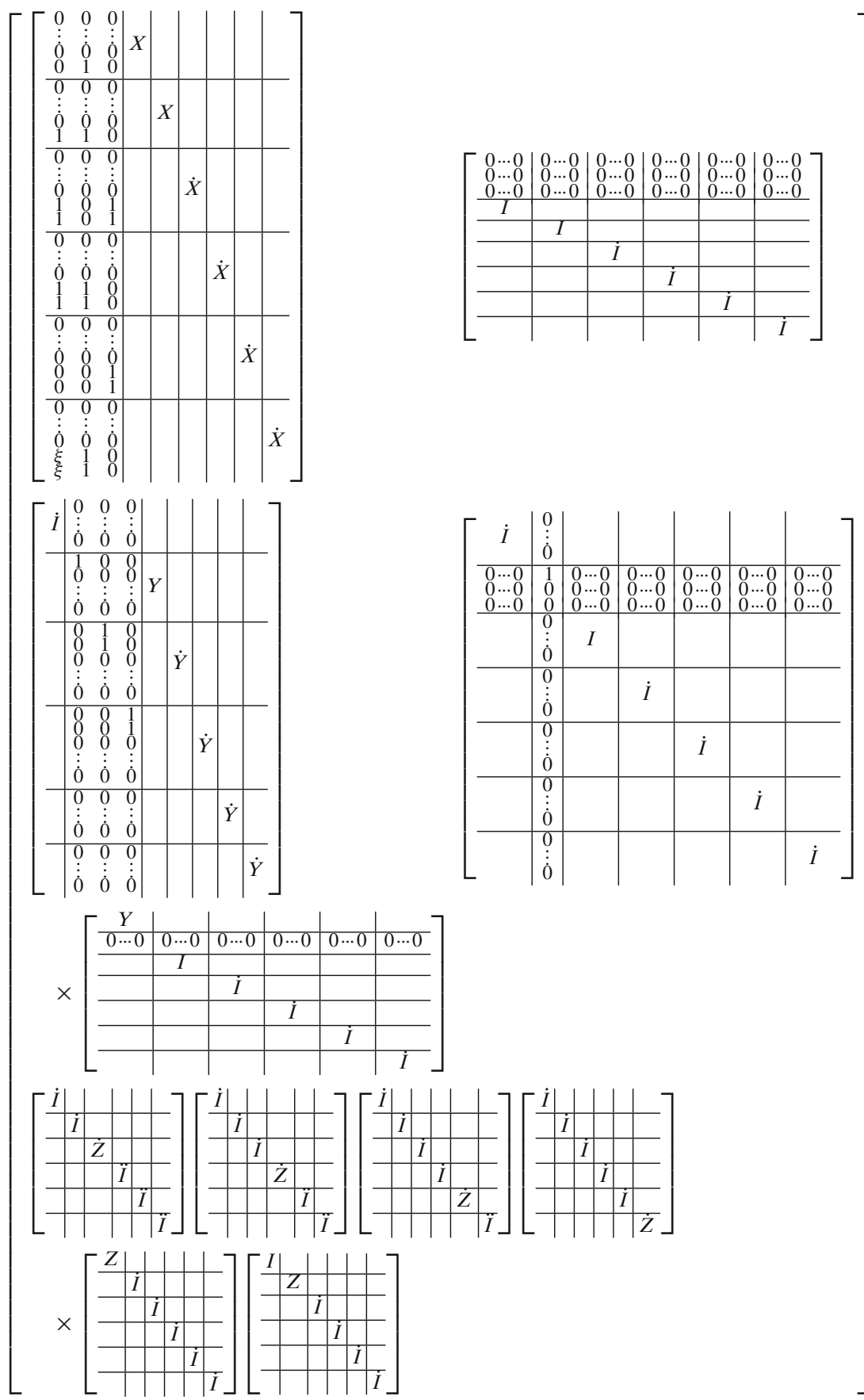
- $\quad[2,3,6], r=5 ; \bar{n}=6 m+5$ :

$\left[\begin{array}{llllll}(m+1)+5(m+2) & & 3+m+5(m+1) & & & \\ & & (m+1)+3+5(m+1) & & & \\ & 2(m+1)+4(m+2) & 3(m+1)+3(m+2) & 4(m+1)+2(m+2) & 5(m+1)+(m+2) & 6(m+1)\end{array}\right]$
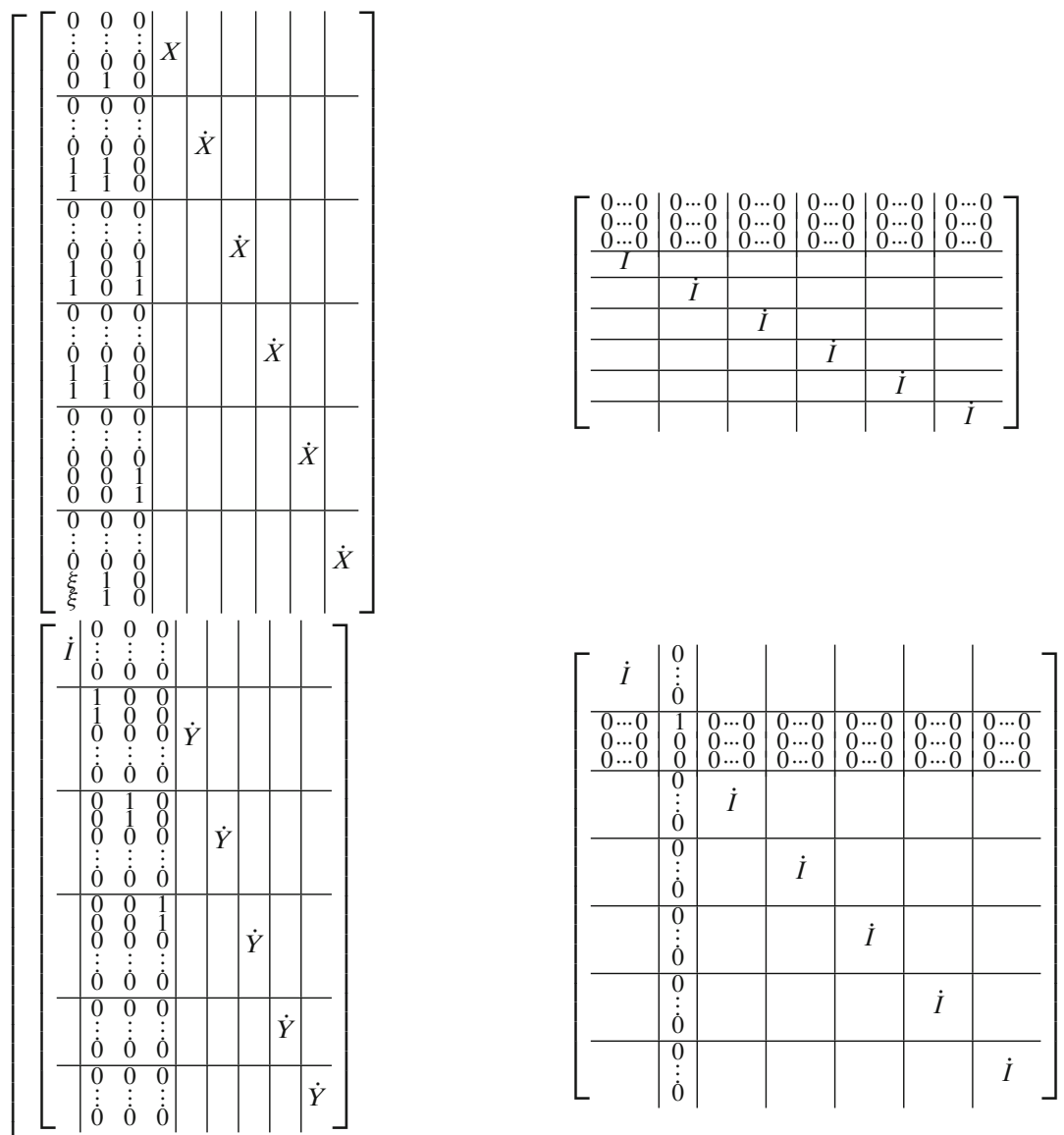

$\times\left[\begin{array}{c|c|c|c|c|c}Y & & & & & \\ \hline 0 \cdots 0 & 0 \cdots 0 & 0 \cdots 0 & 0 \cdots 0 & 0 \cdots 0 & 0 \cdots 0 \\ \hline & \dot{I} & \dot{I} & & & \\ \hline & & & \dot{I} & & \\ \hline & & & & \dot{I} & \\ \hline & & & & \end{array}\right]$
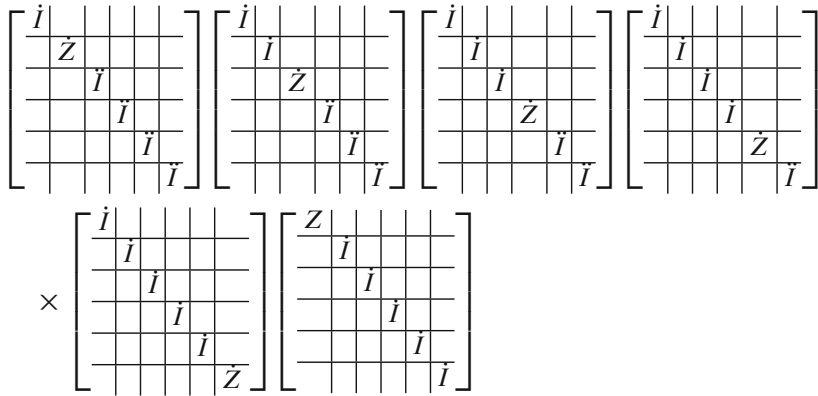
The symbol $\times$ in the last six arrays always denotes the continuation of the row above.

Final Remark Independently of the proof, all the bimodules $B^{(n)}$ from the table above have passed detailed tests in order to make sure we did not make any miscalculations during a whole long and complicated process of their construction. In particular, we checked that the specializations $B^{(n)}(c, l):=k[\xi] /(\xi-c)^{l} \otimes_{k[\xi]} B^{(n)}$, for $(c, l) \in\left(k \backslash \mathcal{Z}\left(f_{\mathbf{p}}\right)\right) \times \mathbb{N}_{\geq 1}$ indeed:

- have appropriate dimension vectors,

- are indecomposable (their endomorphism algebras turned out to be local),

- $\quad$ are Hom-orthogonal (i.e. for fixed slope $n, \operatorname{Hom}_{\Lambda}\left(B^{(n)}(c, l), B^{(n)}\left(c^{\prime}, l^{\prime}\right)\right)=0$ for any $c, c^{\prime} \in k \backslash \mathcal{Z}\left(f_{\mathbf{p}}\right), c \neq c^{\prime}$ and $\left.l, l^{\prime} \geq 1\right)$,

(cf. Remark 4.1(a)).

Open Access This article is distributed under the terms of the Creative Commons Attribution License which permits any use, distribution, and reproduction in any medium, provided the original author(s) and the source are credited.

\section{References}

1. Assem, I., Simson, D., Skowroński, A.: Elements of Representation Theory of Associative Algebras, vol. 1. Techniques of Representation Theory, London Math. Soc. Student Texts 65. Cambridge University Press, Cambridge (2006)

2. Crawley-Boevey, W.W.: Tame algebras and generic modules. Proc. Lond. Math. Soc. 63(3), 241265 (1991)

3. Dlab, V., Ringel, C.M.: Indecomposable representations of graphs and algebras. Mem. Am. Math. Soc. 173(6), (1976)

4. Dowbor, P., Mróz, A.: The multiplicity problem for indecomposable decompositions of modules over domestic canonical algebras. Colloq. Math. 111(2), 221-282 (2008)

5. Dowbor, P., Mróz, A.: On a separation of orbits in the module variety for domestic canonical algebras. Colloq. Math. 111(2), 283-295 (2008)

6. Dowbor, P., Mróz, A.: On a separation of orbits in the module variety for special biserial algebras. J. Pure Appl. Algebra 213(9), 1804-1815 (2009)

7. Dowbor, P., Skowroński, A.: On the representation type of locally bounded categories. Tsukuba J. Math. 10, 63-72 (1986)

8. Dowbor, P., Meltzer, H., Mróz, A.: An algorithm for the construction of exceptional modules over tubular canonical algebras. J. Algebra 323(10), 2710-2734 (2010)

9. Gantmacher, F.R.: Matrix Theory. Chelsea, New York (1959)

10. Geigle, W., Lenzing, H.: A class of weighted projective curves arising in representation theory of finite dimensional algebras. In: Singularities, Representations of Algebras, and Vector Bundles. Lecture Notes in Mathematics, vol. 1273, pp. 265-297. Springer (1987)

11. Happel, D.: Triangulated categories in the representation theory of finite dimensional algebras. In: London Math. Soc. Lecture Notes Series, vol. 119 (1988)

12. Hartshorne, R.: Residues and duality. In: Lecture Notes Math., vol. 20. Springer, Berlin (1966)

13. D. Kędzierski, D., Meltzer, H.: Indecomposable represenations for extended Dynkin quivers of type $\tilde{\mathbb{E}}_{8}$. Colloq. Math. 111(1), 95-116 (2011)

14. Kostrikin, A.I.: Introduction to Algebra. Springer, Berlin (1982)

15. Kussin, D., Meltzer, H.: Indecomposable modules for domestic canonical algebras. J. Pure Appl. Algebra 211, 471-483 (2007) 
16. Lenzing, H., Meltzer, H.: Sheaves on a weighted projective line of genus one and representations of a tubular algebra. In: Representations of Algebras, Sixth International Conference, Ottawa 1992. CMS Conf. Proc., vol. 14, pp. 317-337 (1993)

17. Meltzer, H.: Exceptional modules for tubular canonical algebras. Algebr. Represent. Theory 10, 481-496 (2007)

18. Ringel,C.M.: Tame algebras and integral quadratic forms. In: Lecture Notes in Mathematics, vol. 1099. Springer (1984)

19. Simson, D., Skowroński, A.: Elements of the Representation Theory of Associative Algebras, vol. 3. Representation-Infinite Tilted Algebras. London Math. Soc. Student Texts, vol. 71. Cambridge Univ. Press, Cambridge (2007)

20. Smith, H.J.S.: On systems of linear indeterminate equations and congruences. In: Collected Math. Papers, vol. 1, pp. 367-409. Chelsea (1979) 